\title{
Extended dynamically weighted CASPT2: the best of two worlds
}

\author{
Stefano Battaglia* and Roland Lindh* \\ Department of Chemistry - BMC, Uppsala University, P.O. Box 576, SE-75123 Uppsala, \\ Sweden \\ E-mail: stefano.battaglia@kemi.uu.se; roland.lindh@kemi.uu.se
}

\section{Abstract}

We introduce a new variant of the complete active space second-order perturbation theory (CASPT2) method that performs similarly to multistate CASPT2 (MS-CASPT2) in regions of the potential energy surface where the electronic states are energetically well separated and is akin to extended MS-CASPT2 (XMSCASPT2) in case the underlying zeroth-order references are near-degenerate. Our approach follows a recipe analogous to XMS-CASPT2 to ensure approximate invariance under unitary transformations of the model states and a dynamical weighting scheme to smoothly interpolate the Fock operator between a statespecific and a state-average regime. The resulting extended dynamically weighted CASPT2 (XDW-CASPT2) methodology possesses the most desirable features of both MS-CASPT2 and XMS-CASPT2, i.e. the ability to provide accurate transition energies and correctly describe avoided crossings and conical intersections. The reliability of XDW-CASPT2 is assessed on a number of molecular systems. First, we consider the dissociation of lithium fluoride, highlighting the distinctive characteristics of the new approach. Second, the invariance of the theory is investigated by studying the conical intersection of the distorted allene molecule. Finally, the relative accuracy in the calculation of vertical excitation energies is benchmarked on a set of 26 organic compounds. We found that XDW-CASPT2, al- beit being only approximately invariant, produces smooth potential energy surfaces around conical intersections and avoided crossings, performing equally well to the strictly invariant XMS-CASPT2 method. The accuracy of vertical transition energies is almost identical to MS-CASPT2, with a mean absolute deviation of 0.01 to $0.02 \mathrm{eV}$, in contrast to $0.12 \mathrm{eV}$ for XMS-CASPT2.

\section{Introduction}

The theoretical modeling of excited states processes is undoubtedly of fundamental and practical importance. ${ }^{1}$ The investigation of physical mechanisms at the base of chemi- and bioluminescence, ${ }^{2}$ spectroscopy,${ }^{3}$ singlet fission, ${ }^{4}$ and many other scientifically and technologically relevant applications require methodologies that are able to describe the entire potential energy surface (PES), providing accurate relative energies between different electronic states, their correct orderings and the right morphology in regions of neardegeneracies. ${ }^{5}$ Single-reference approaches, despite their widespread success, do not generally have the necessary flexibility to be applied indistinctly in any situation that one might encounter in the realm of excited states chemistry: a multireference approach is unavoidable. From the several available options, multireference perturbation theory (MRPT) stands out: its accuracy, general applicability and moderate computational cost elected it during the 
last few decades as the method of choice for the investigation of full potential energy surfaces. ${ }^{5}$ In particular, formalisms that allow the relaxation of the reference states under the influence of the perturbation have seen the most success, ${ }^{6-20}$ with the multistate complete active space second-order perturbation theory (MSCASPT2 $)^{11}$ approach being one of the most popular. Relying on the multipartitioning technique, ${ }^{8}$ this methodology is well suited for the calculation of transition energies between states that are well separated, with deviations within 0.1 to $0.2 \mathrm{eV}$ from the best theoretical estimates. ${ }^{21,22}$ On the other hand, even though MS-CASPT2 follows the "diagonalizethen-perturb-then-diagonalize" philosophy, it may still suffer from unphysical behaviors at molecular geometries with near-degenerate reference states. The theoretical understanding of this shortcoming is known ${ }^{23}$ and it can be solved by enforcing the states to be invariant under unitary transformations within the model space, leading to the so-called extended MSCASPT2 (XMS-CASPT2) ${ }^{24}$ method. However, the latter requires a unique partitioning of the Hamiltonian achieved through the use of a state-average Fock operator, which is likely to degrade the accuracy as the dimension of the model space is increased or when the states under consideration are of different character (e.g. valence and Rydberg).

The main objective of this work is to formulate a new CASPT2 variant that retains the accuracy of MS-CASPT2 in the calculation of transition energies and at the same time yields smooth potential energy surfaces with no artifacts in regions where the excited states manifold is near-degenerate. Our approach is based on the same transformation carried out in the initial step of XMS-CASPT2 and then uses a dynamical weighting scheme to interpolate between state-specific and state-average operators; hence we call it extended dynamically weighted CASPT2 (XDW-CASPT2). From a theoretical standpoint, XDW-CASPT2 corresponds to a new and somewhat sophisticated partitioning of the Hamiltonian, thus retaining the underlying structure of the parent theory. Recently, an analogous attempt to find a zeroth-order Hamiltonian that coincides with the canonical MS-CASPT2 one, but that at the same time is invariant as in XMS-CASPT2 was carried out by Park ${ }^{25}$. XDW-CASPT2 also shares some similarities with the recently introduced dynamically weighted driven similarity renormalization group (DW-DSRG) ${ }^{26}$ as well as the dynamically weighted complete active space self-consisted field method. ${ }^{27,28}$

The article is structured as follows. In section 2 we first selectively review important aspects of quasidegenerate perturbation theory (QDPT), MS-CASPT2 and XMS-CASPT2 necessary to define XDW-CASPT2 in the remainder of the section. Next, section 3 is devoted to the assessment of the new methodology and is divided in three parts. First, an extensive study on the dissociation of LiF is presented, being this problem a prototypical example to show all features of the new method. Second, the conical intersection in the distorted allene molecule is investigated, which represents a difficult case for QDPT-based approaches. Third, the accuracy of vertical transition energies to the lowest singlet excited state is evaluated on a set of 26 small to medium organic compounds. At last, in section 4 we conclude by summarizing the results obtained in this contribution and with an outlook on future directions regarding XDW-CASPT2.

\section{Theory}

As in any perturbation theory approach, the starting point is to partition the full Hamiltonian into a zeroth-order part $\hat{H}_{0}$, with known eigenfunctions $\Psi_{\alpha}^{(0)}$ and associated eigenvalues $E_{\alpha}^{(0)}$, and a perturbation operator $\hat{V}=\hat{H}-\hat{H}_{0}$. The Hilbert space is also partitioned into a model space, spanned by model functions (also called reference functions) selected from the set of zeroth-order ones, and a complementary space, spanned by all other functions orthogonal to the model ones. The projector onto the model space is defined as

$$
\hat{P}=\sum_{\gamma \in \mathcal{P}}\left|\Psi_{\gamma}^{(0)}\right\rangle\left\langle\Psi_{\gamma}^{(0)}\right|
$$


where $\mathcal{P}$ denotes the set of indices labeling the reference states. The projector onto the complementary space is simply defined as $\hat{Q}=$ $\hat{1}-\hat{P}$. Note that the complementary space does not necessarily have to be spanned by the remaining zeroth-order functions not included in the model space: other types of many-electron functions can be used. The wave operator ${ }^{29} \hat{\Omega}$, defined as an operator that acting on a model state $\Psi_{\alpha}^{(0)}$ generates the exact one (i.e. of the full Hamiltonian $\hat{H}$ )

$$
\hat{\Omega} \Psi_{\alpha}^{(0)}=\Psi_{\alpha}
$$

is governed by the generalized Bloch equation ${ }^{30}$

$$
\left[\hat{\Omega}, \hat{H}_{0}\right]=\hat{Q} \hat{V} \hat{\Omega}-\hat{Q} \hat{\Omega} \hat{V} \hat{\Omega}
$$

Assuming intermediate normalization, an effective Hamiltonian is constructed according to

$$
\hat{H}_{e f f}=\hat{P} \hat{H} \hat{\Omega} \hat{P}
$$

whose eigenvalues and eigenfunctions (within the model space) correspond to the exact ones. To arrive at a practical implementation of QDPT, $\hat{\Omega}$ is expanded in powers of the perturbation operator

$$
\hat{\Omega}=\hat{\Omega}^{(0)}+\hat{\Omega}^{(1)}+\hat{\Omega}^{(2)}+\ldots
$$

and substituted into Equation (4) leading to the second-order effective Hamiltonian

$$
\hat{H}_{e f f}^{(2)}=\hat{P} \hat{H} \hat{\Omega}^{(1)} \hat{P}
$$

with the superscript $(n)$ denoting the order in $\hat{V}$. Expressing $\hat{H}_{\text {eff }}^{(2)}$ in the model space basis and diagonalizing the resulting matrix provides the second-order correction to the energies and the perturbatively modified zerothorder wave functions. To determine $\hat{\Omega}^{(1)}$ in Equation (6), one has to solve the first-order generalized Bloch equation

$$
\left[\hat{\Omega}^{(1)}, \hat{H}_{0}\right]=\hat{Q} \hat{V} \hat{P}
$$

which is obtained upon inserting Equation (5) into Equation (3) and equating only the terms which are of first order in $\hat{V}$. Note that the application of $\hat{\Omega}^{(1)}$ to $\Psi_{\alpha}^{(0)}$ generates the first- order correction to the wave function

$$
\Psi_{\alpha}^{(1)}=\hat{\Omega}^{(1)} \Psi_{\alpha}^{(0)}
$$

for all $\alpha \in \mathcal{P}$.

\section{$2.1 \quad$ MS-CASPT2}

In the multistate CASPT2 method $^{11}$ the zeroth-order functions defining the model space are of complete active space self-consistent field (CASSCF) type. For each $\alpha \in \mathcal{P}$, there is a separate partitioning of the full Hamiltonian, ${ }^{31}$ $\hat{H}=\hat{H}_{0}^{\alpha}+\hat{V}^{\alpha}$, with the zeroth-order part defined by

$$
\begin{aligned}
\hat{H}_{0}^{\alpha} & =\sum_{\gamma \in \mathcal{P}}\left|\Psi_{\gamma}^{(0)}\right\rangle\left\langle\Psi_{\gamma}^{(0)}\left|\hat{f}^{\alpha}\right| \Psi_{\gamma}^{(0)}\right\rangle\left\langle\Psi_{\gamma}^{(0)}\right| \\
& +\sum_{k \in \mathcal{P} \perp}\left|\Psi_{k}^{(0)}\right\rangle\left\langle\Psi_{k}^{(0)}\left|\hat{f}^{\alpha}\right| \Psi_{k}^{(0)}\right\rangle\left\langle\Psi_{k}^{(0)}\right| \\
& +\hat{Q}_{S D} \hat{f}^{\alpha} \hat{Q}_{S D}+\hat{Q}_{T Q \ldots} \hat{f}^{\alpha} \hat{Q}_{T Q \ldots}
\end{aligned}
$$

The first sum is restricted to states in the model space (including $\gamma=\alpha$ ), while the second one runs over all other states of the complete active space, with $\mathcal{P}^{\perp}$ being the set of indices labeling them. The remaining part of the complementary space is spanned by internally contracted configurations (ICCs) obtained by the application of excitation operators to the reference states $\Psi_{\alpha}^{(0)}$. The operator $\hat{Q}_{S D}$ projects onto the so-called first-order interacting space that, for the sake of this theoretical discussion, we shall assume is always generated from the union of all model states ${ }^{1}$. Similarly, $\hat{Q}_{T Q \ldots}$ projects onto the space spanned by higher-order ICCs. The generalized Fock operator $\hat{f}^{\alpha}$ is given by

$$
\hat{f}^{\alpha}=\sum_{p q} f_{p q}^{\alpha} \hat{E}_{p q}
$$

where $\hat{E}_{p q}$ is the second-quantized spin-summed one-particle excitation operator and $f_{p q}^{\alpha}$ are en-

\footnotetext{
${ }^{1}$ This seemingly insignificant choice avoids a discussion on single-state single-reference (SS-SR) and multistate multireference (MS-MR) variants of internally contracted theories which is not the primary focus of this work. For a thorough comparison between them in the context of CASPT2 see e.g. the recent work by Park ${ }^{25}$.
} 
tries of the Fock matrix expressed in the molecular orbital basis

$$
f_{p q}^{\alpha}=h_{p q}+\sum_{r s} D_{r s}^{\alpha}\left[(p q \mid r s)-\frac{1}{2}(p r \mid q s)\right]
$$

Here, $h_{p q}$ and $(p q \mid r s)$ are elements of the oneparticle Hamiltonian and the two-electron repulsion integrals, respectively, while $D_{r s}^{\alpha}=$ $\left\langle\Psi_{\alpha}^{(0)}\left|\hat{E}_{r s}\right| \Psi_{\alpha}^{(0)}\right\rangle$ are entries of the one-particle reduced density matrix (1-RDM, or also simply called density matrix) of state $\Psi_{\alpha}^{(0)}$. The indices $p, q, r$ and $s$ label general molecular orbitals. The use of projectors in Equation (9) for the definition of $\hat{H}_{0}$ is necessary because the CASSCF states are not eigenfunctions of the generalized Fock operator. Furthermore, note that $\hat{H}_{0}$ is diagonal within the model space since $\hat{f}^{\alpha}$ is projected directly onto the reference states rather than onto the space spanned by them. In other words, even though in general

$$
\left\langle\Psi_{\alpha}^{(0)}\left|\hat{f}^{\gamma}\right| \Psi_{\beta}^{(0)}\right\rangle \neq 0
$$

for $\alpha \neq \beta \in \mathcal{P}$ and $\gamma \in \mathcal{P}$, these elements are arbitrarily set to zero in the MS-CASPT2 $\hat{H}_{0}$. This constitutes an approximation that we will call hereafter diagonal approximation. The immediate consequence of this choice is that upon inserting Equation (9) into Equation (7), the solution of the first-order generalized Bloch equation can be obtained for each state of the model space separately, as these are not coupled anymore. The substantial advantage gained is the possibility to use state-specific Fock operators in $\hat{H}_{0}$, allowing for a formalism based on multipartitioning that should provide more accurate zeroth-order energies. In particular, for states that are energetically well separated or have considerably different character, state-specific Fock operators are in principle better suited to describe them than, for instance, a single operator that requires the flexibility to account for all states in an average way.

On the other hand, the diagonal approximation has a profound impact on the invariance properties of the method as elucidated by Granovsky $^{23}$ in the context of multiconfigurational QDPT (MCQDPT). The main issues are two. First, when two reference states interact strongly at zeroth-order, meaning that the element $\left\langle\Psi_{\alpha}^{(0)}\left|\hat{f}^{\gamma}\right| \Psi_{\beta}^{(0)}\right\rangle$ is significantly larger than zero, it can be shown ${ }^{23}$ that neglecting it leads to large systematic errors in the corresponding off-diagonal element of the second-order effective Hamiltonian. Second, it is known that zeroth-order states at a conical intersection (CI), and to a large extent at an avoided crossing (AC) as well, are not well-defined: in such situations any linear superposition of the involved states constitutes an equally valid wave function. Hence, simply projecting the Fock operator onto the individual components entails an arbitrary choice which might lead to the appearance of artifacts on the potential energy surface in the vicinity of the AC or CI.

In conclusion, we should note that it is possible to adopt a unique partitioning in MSCASPT2, for instance with the use of a stateaverage Fock operator. Such an approach however would lose the advantages of multipartitioning, but keep the issues related to the lack of invariance. Nevertheless, this strategy has been recently explored by Kats and Werner ${ }^{32}$ in the context of pair natural orbital MS-CASPT2, finding systematic deviations from canonical MS-CASPT2 by 0.1 to $0.2 \mathrm{eV}$ for transitions to the lowest singlet excited state.

\subsection{XMS-CASPT2}

The main flaw of MS-CASPT2 is the lack of invariance under unitary transformations within the model space. The result obtained with a particular set of reference states should always be the same to the one obtained with a set of states generated by a unitary transformation of the original ones. This shortcoming is ascribed to the diagonal approximation of $\hat{H}_{0}$ and the solution to this problem was first proposed by Granovsky $^{23}$ for MCQDPT and shortly after applied to MS-CASPT2 by Shiozaki et al. ${ }^{24}$. The key difference of the new methodology, XMS-CASPT2, is in the zeroth-order Hamilto- 
nian

$$
\begin{aligned}
& \hat{H}_{0}=\sum_{\gamma, \delta \in \mathcal{P}}\left|\Psi_{\gamma}^{(0)}\right\rangle\left\langle\Psi_{\gamma}^{(0)}\left|\hat{f}^{s a}\right| \Psi_{\delta}^{(0)}\right\rangle\left\langle\Psi_{\delta}^{(0)}\right| \\
& +\sum_{k \in \mathcal{P}^{\perp}}\left|\Psi_{k}^{(0)}\right\rangle\left\langle\Psi_{k}^{(0)}\left|\hat{f}^{s a}\right| \Psi_{k}^{(0)}\right\rangle\left\langle\Psi_{k}^{(0)}\right| \\
& +\hat{Q}_{S D} \hat{f}^{s a} \hat{Q}_{S D}+\hat{Q}_{T Q \ldots} \hat{f}^{s a} \hat{Q}_{T Q \ldots}
\end{aligned}
$$

The Fock operator is now projected onto the full model space rather than on the individual components alone. This implies a unique partitioning of the Hamiltonian because the first-order generalized Bloch equation, Equation (7), does not decouple the states anymore. The Fock operator $\hat{f}^{s a}$ is constructed from the state-average density matrix

$$
\mathbf{D}^{s a}=\frac{1}{d} \sum_{\alpha \in \mathcal{P}} \mathbf{D}^{\alpha}
$$

for a model space containing $d$ states $^{2}$. The fact that $\hat{H}_{0}$ is no longer diagonal in the zeroth-order basis makes the solution of Equation (7) somewhat harder. However, this complication can be fully overcome by a unitary transformation of the reference states, such that the rotated wave functions

$$
\tilde{\Psi}_{\alpha}^{(0)}=\sum_{\beta \in \mathcal{P}} U_{\beta \alpha} \Psi_{\beta}^{(0)}
$$

diagonalize the Fock operator within the model space. In other words, the rotated model states satisfy

$$
\left\langle\tilde{\Psi}_{\alpha}^{(0)}|\hat{f} s a| \tilde{\Psi}_{\beta}^{(0)}\right\rangle=0
$$

for $\alpha \neq \beta \in \mathcal{P}$. Using the wave functions $\tilde{\Psi}_{\alpha}^{(0)}$, the zeroth-order Hamiltonian can now be rewritten as

$$
\begin{aligned}
& \hat{H}_{0}=\sum_{\gamma \in \mathcal{P}}\left|\tilde{\Psi}_{\gamma}^{(0)}\right\rangle\left\langle\tilde{\Psi}_{\gamma}^{(0)}\left|\hat{f}^{s a}\right| \tilde{\Psi}_{\gamma}^{(0)}\right\rangle\left\langle\tilde{\Psi}_{\gamma}^{(0)}\right| \\
& +\sum_{k \in \mathcal{P} \perp}\left|\Psi_{k}^{(0)}\right\rangle\left\langle\Psi_{k}^{(0)}\left|\hat{f}^{s a}\right| \Psi_{k}^{(0)}\right\rangle\left\langle\Psi_{k}^{(0)}\right| \\
& +\hat{Q}_{S D} \hat{f}^{s a} \hat{Q}_{S D}+\hat{Q}_{T Q \ldots} \hat{f}^{s a} \hat{Q}_{T Q \ldots}
\end{aligned}
$$

${ }^{2}$ Note that using the state-average density matrix to construct the Fock operator or averaging the statespecific Fock operators lead to the same $\hat{f}^{s a}$. which has the same form of Equation (9), albeit the use of the state-average Fock operator. Therefore, MS-CASPT2 truly corresponds to an approximation of XMS-CASPT2, provided that the same unique partitioning of the Hamiltonian is used in both variants. The generalizations introduced with Equation (13) make this method invariant under unitary transformations of the model space wave functions, solving the issues intrinsic to the diagonal approximation of MS-CASPT2. As a result, XMSCASPT2 is more robust in general, with energies that are continuous and smooth functions of the molecular geometry even in the vicinity of ACs and CIs. The price to pay is the use of $\hat{f}^{s a}$ in $\hat{H}_{0}$, which, as briefly discussed at the end of the previous subsection, is likely to decrease the accuracy of the method for the calculation of excitation energies, in particular when these are large or between states of different character. Nevertheless, to the best of our knowledge, no systematic and comprehensive benchmark on the accuracy of XMS-CASPT2 is available in the literature.

\subsection{XDW-CASPT2}

The necessary ingredients to design a hybrid approach that interpolates between MS-CASPT2 and XMS-CASPT2 are the use of state-specific Fock operators in a multipartitioning formalism and the projection of $\hat{H}_{0}$ onto the full model space rather than onto individual reference states. The objective is a method that performs as well as MS-CASPT2 in situations where states are clearly discernible and is as robust as XMS-CASPT2 when these are instead quasidegenerate. We note from our previous discussion that in case the zeroth-order Hamiltonian has negligible off-diagonal elements within the model space, i.e.

$$
\left\langle\Psi_{\alpha}^{(0)}\left|\hat{H}_{0}\right| \Psi_{\beta}^{(0)}\right\rangle \approx 0
$$

the diagonal approximation is a sound simplification of the generalized Bloch equation. Crucially, it allows for a formalism based on multipartitioning. Thus, we are seeking a unitary transformation as in Equation (15), whereby 
the rotated states satisfy

$$
\left\langle\tilde{\Psi}_{\alpha}^{(0)}|\hat{\bar{f}} \gamma| \tilde{\Psi}_{\beta}^{(0)}\right\rangle \approx 0
$$

for $\alpha \neq \beta \in \mathcal{P}$, with the Fock operator $\hat{\bar{f}}^{\gamma}$ (note the bar to differentiate this operator from the normal state-specific one) having the following property

$$
\hat{\bar{f}}^{\gamma} \approx \begin{cases}\hat{f}^{\gamma} & \text { if } \tilde{\Psi}_{\gamma}^{(0)} \text { weakly interacts } \\ & \text { with other model states } \\ \hat{f}^{s a} & \text { if } \tilde{\Psi}_{\gamma}^{(0)} \text { strongly interacts } \\ & \text { with other model states }\end{cases}
$$

for all $\gamma \in \mathcal{P}$. Note that we shall better specify further below what does weak and strong interaction mean in this context.

We are able to satisfy Equations (19) and (20) with the following scheme. In a first step, completely analogous to XMS-CASPT2, a set of rotated model states $\widetilde{\Psi}_{\alpha}^{(0)}$ is obtained by diagonalization of the state-average Fock operator $\hat{f}^{s a}$. These functions are then used to construct dynamically weighted density matrices of the form

$$
\overline{\mathbf{D}}^{\alpha}=\sum_{\beta \in \mathcal{P}} \omega_{\alpha}^{\beta} \tilde{\mathbf{D}}^{\beta}
$$

with weights satisfying the condition

$$
\sum_{\beta \in \mathcal{P}} \omega_{\alpha}^{\beta}=1
$$

for all $\alpha \in \mathcal{P}$. The use of tildes emphasizes that $\tilde{\mathbf{D}}^{\beta}$ is the $1-\mathrm{RDM}$ associated to the rotated state $\tilde{\Psi}_{\beta}^{(0)}$. Using the densities defined in Equation (21), state-specific Fock operators are constructed according to Equations (10) and (11) for all $\alpha \in \mathcal{P}$ and used to define the partitionings of the Hamiltonian for a subsequent MSCASPT2 calculation. Thus, XDW-CASPT2 substantially consists in a MS-CASPT2 calculation employing zeroth-order states defined by Equation (15) and state-specific Fock operators constructed with densities $\overline{\mathbf{D}}^{\alpha}$.

The weights $\omega_{\alpha}^{\beta}$ are chosen such that the resulting Fock operators $\hat{\bar{f}}^{\alpha}$ satisfy the prescription of Equation (20). This is achieved by using a scheme recently introduced by one of the authors of this contribution and his collaborators, ${ }^{26}$ whereby $\omega_{\alpha}^{\beta}$ is defined by the following Boltzmann-like function

$$
\omega_{\alpha}^{\beta}=\frac{e^{-\zeta\left(\Delta_{\alpha \beta}\right)^{2}}}{\sum_{\gamma \in \mathcal{P}} e^{-\zeta\left(\Delta_{\alpha \gamma}\right)^{2}}}
$$

where $\Delta_{\alpha \beta}\left(\Delta_{\alpha \gamma}\right)$ quantifies the interaction between states $\tilde{\Psi}_{\alpha}^{(0)}$ and $\tilde{\Psi}_{\beta}^{(0)}\left(\tilde{\Psi}_{\gamma}^{(0)}\right)$ and $\zeta \in \mathbb{R}_{0}^{+}$ is a parameter controlling the sharpness of the transition between a mixed-density and a statespecific regime. Let us list the asymptotic properties of Equation (23) with respect to $\Delta_{\alpha \beta}$ :

$$
\begin{aligned}
& \Delta_{\alpha \beta} \rightarrow \infty \Longrightarrow \omega_{\alpha}^{\beta} \rightarrow 0 \\
& \Delta_{\alpha \beta} \rightarrow 0 \Longrightarrow \omega_{\alpha}^{\beta}=\omega_{\beta}^{\alpha}
\end{aligned}
$$

A physical quantity that satisfies Equations (24) and (25) is given by the energy difference between the rotated states

$$
\Delta_{\alpha \beta}=\left|\left\langle\tilde{\Psi}_{\alpha}^{(0)}|\hat{H}| \tilde{\Psi}_{\alpha}^{(0)}\right\rangle-\left\langle\tilde{\Psi}_{\beta}^{(0)}|\hat{H}| \tilde{\Psi}_{\beta}^{(0)}\right\rangle\right|
$$

When computing the contribution of state $\Psi_{\beta}^{(0)}$ to the density of $\Psi_{\alpha}^{(0)}$, if their energy difference is large $-\Delta_{\alpha \beta} \gg 0$ - then $\Psi_{\beta}^{(0)}$ should not contribute: $\omega_{\alpha}^{\beta} \approx 0$. This situation corresponds to the case in which the two states are weakly or not interacting. Vice-versa, if the energy difference is small $-\Delta_{\alpha \beta} \approx 0-$ then $\Psi_{\beta}^{(0)}$ is quasidegenerate with $\Psi_{\alpha}^{(0)}$ and should receive approximately the same weight: $\omega_{\alpha}^{\beta} \approx \omega_{\beta}^{\alpha}$. This situation corresponds to the case in which the two states are strongly interacting. Note that, somewhat counterintuitively, strong interaction is associated with a small value of the parameter $\Delta_{\alpha \beta}$ and conversely weak interaction with a large one. Simply using an energetic criterion to parametrize the interaction strength between two states can lead to unphysical averaging: e.g. two states of different symmetry (spin or spatial) should not be mixed together irrespective of their relative energy. In this work, this problem has been circumvented by treating states of different symmetry separately, however, a more general solution is possible. For instance, by multiplying the right-hand side of Equation (26) by a factor dependent 
on the off-diagonal element of the full Hamiltonian expressed in the basis of rotated references, $\left\langle\tilde{\Psi}_{\alpha}^{(0)}|\hat{H}| \tilde{\Psi}_{\beta}^{(0)}\right\rangle, \Delta_{\alpha \beta}$ would account for the physical nature of the states without resorting on external constraints (e.g. forcing symmetries). Importantly, such a modification would correctly model changes of the molecular geometry that break the symmetry of the system.

For a fixed state $\Psi_{\alpha}^{(0)}$, the parameter $\zeta$ modulates the importance of other states in a collective manner: a small value tends to make them all equally important, whereas a large value favors the parent state under consideration. The asymptotic behavior of $\omega_{\alpha}^{\beta}$ with respect to $\zeta$ is given by

$$
\begin{gathered}
\zeta \rightarrow \infty \Longrightarrow \begin{cases}\omega_{\alpha}^{\beta} \rightarrow 0 & \text { if } \beta \neq \alpha \\
\omega_{\alpha}^{\beta} \rightarrow 1 & \text { if } \beta=\alpha\end{cases} \\
\zeta \rightarrow 0 \Longrightarrow \omega_{\alpha}^{\beta} \rightarrow \frac{1}{d} \quad \forall \beta \in \mathcal{P}
\end{gathered}
$$

where $d$ is the number of model states. The situation depicted in Equation (27) results in purely state-specific Fock operators alike MSCASPT2, albeit using densities of rotated reference functions. On the other hand, if all weights are equal as shown in Equation (28), the original XMS-CASPT2 is restored. For the particular choice of $\Delta_{\alpha \beta}$ made in Equation (26), $\zeta$ assumes $E_{\mathrm{h}}^{-2}$ units and its value can be regarded as a threshold. When the value of $\Delta_{\alpha \beta}$ is in the same order of magnitude as $\zeta^{-1 / 2}$ or smaller, the state $\Psi_{\beta}^{(0)}$ will contribute significantly to $\hat{\bar{f}}^{\alpha}$, if instead $\Delta_{\alpha \beta} \gg \zeta^{-1 / 2}$, it will play little to no role in $\hat{\bar{f}}^{\alpha}$.

As a final remark, we should note that Equation (23) was used in a similar fashion in the recent work by $\mathrm{Li}$ et al. ${ }^{26}$, where not only the 1-RDM was averaged with dynamical weights, but also higher-order RDMs. Importantly, the latter were introduced in the flow equations, whose solution provides the diagonal matrix elements of the effective Hamiltonian. In the approach presented here, the densities defined by Equation (21) are only used to obtain an alternative partitioning of the Hamiltonian; the first-order equations that determine the correction to the wave function, and accordingly $\hat{H}_{e f f}$, make use of purely state-specific densities.

\section{Results}

In this section we present the results obtained for a series of calculations representing typical use-case scenarios in order to assess the reliability of XDW-CASPT2. First, the avoided crossings in $\mathrm{LiF}$ are investigated. This prototypical system is an ideal model to highlight the strengths, weaknesses and features of XDW-CASPT2 as compared to MS-CASPT2 and XMS-CASPT2. Since this example touches every aspect of the theory, the discussion of this case is quite extensive. Second, the conical intersection in the distorted allene molecule is considered. This system provides a tougher test for the invariance properties of the theory, thereby probing the robustness of the approach. At last, singlet vertical excitation energies are computed for a series of organic compounds in order to evaluate the accuracy of the method and the effect of the dynamical weighting scheme.

All calculations were performed with a development branch of OpenMolcas ${ }^{33}$ based on the master branch, version v18.09-617-g5a96a25e. Note that the CASPT2 implementation of OpenMolcas uses the SS-SR ICC basis, thereby never fully preserving invariance, not even for XMS-CASPT2.

\subsection{Avoided crossings in LiF}

It is well known that during the dissociation of lithium fluoride the two lowest singlet states of ${ }^{1} \Sigma^{+}$symmetry undergo a rapid change of character switching between ionic and covalent. A state-average CASSCF (SA-CASSCF) calculation predicts the avoided crossing at a much shorter distance compared to the reference values (e.g. full configuration interaction) due to the missing dynamical electron correlation. ${ }^{11,34}$ Introduction of the latter in a state-specific manner, for instance through single-state CASPT2, results in an artificial double crossing of the two potential energy curves (PECs), which, alongside other issues 
present in the theory, has been a main motivation for the development of its multistate generalization. Nevertheless, even though MSCASPT2 provides much more satisfactory results, it still faces severe complications at internuclear distances where the underlying reference states are quasidegenerate. This is particularly visible when considering the three lowest ${ }^{1} \Sigma^{+}$states rather than the usual two. Instead, XMS-CASPT2 does not incur in any unphysical behavior irrespective of the number of states, however at the expense of a reduced accuracy in their relative energy at the equilibrium distance. Thus, lithium fluoride is an ideal system to test XDW-CASPT2, and to this end, we calculated its dissociation considering the three lowest singlet states simultaneously.

The reference wave functions were obtained by a SA-CASSCF ${ }^{35}$ calculation using equal weights for all three states and imposing the $\mathrm{C}_{2 v}$ molecular point group symmetry. The active space was composed by six electrons in 2 $a_{1}, 2 b_{1}$ and $2 b_{2}$ orbitals, while the remaining 3 occupied $a_{1}$ orbitals were relaxed during optimization. The cc-pVTZ ${ }^{36}$ and aug-ccpVTZ basis set ${ }^{37}$ were used on lithium and fluorine, respectively. The potential energy curves were computed for internuclear distances between 2.4 and $14 a_{0}$ in steps of $0.2 a_{0}$. The results obtained with the CASSCF method are shown in Figure 1. At an internuclear distance comprised between 6.8 and $7.8 a_{0}$, the ground state wave function quickly changes from an ionic to a covalent character, whereas the opposite happens for the $2^{1} \Sigma^{+}$one. The inclusion of a third state in the calculation plays a little role here: the position of this avoided crossing is slightly shifted to a shorter internuclear distance compared to a 2-state calculation (see Supporting Information for 2-state PECs). From 10.5 to $11.5 a_{0}$, a second avoided crossing between the $2^{1} \Sigma^{+}$and the $3^{1} \Sigma^{+}$states appear, where the character of the $3^{1} \Sigma^{+}$wave function becomes ionic. These quasidegeneracies among the CASSCF states have important consequences on the accuracy and effectiveness of the perturbative approach used to recover the dynamic electron correlation.

To establish a reference, we report in Figure 2

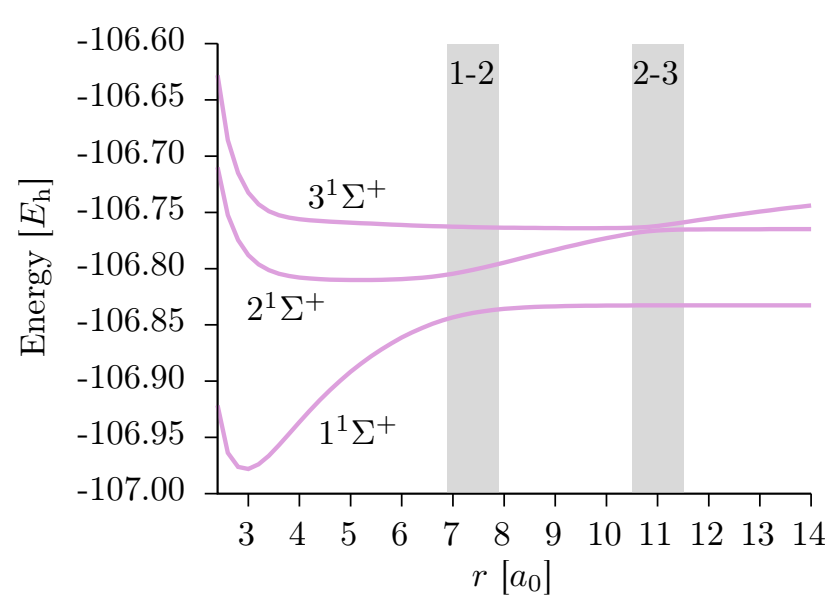

Figure 1: SA-CASSCF potential energy curves of the three lowest ${ }^{1} \Sigma^{+}$states of lithium fluoride. There are two avoided crossing regions (highlighted in gray), one between the ground and the first excited state, labeled 1-2, and one between the first and the second excited state, labeled 2-3.

the PECs computed with MS-CASPT2, XMSCASPT2 and multireference configuration interaction with singles and doubles (MRCISD). For all three methodologies, the two 1s core orbitals were kept frozen, and the 2 s orbital of fluorine was the only doubly-occupied orbital correlated $^{3}$. No shift was used in any CASPT2 calculation: neither real nor imaginary, nor IPEA. In both regions where the avoided crossings happen at CASSCF level, we note a significant, unphysical distortion of the MS-CASPT2 curves, but not for the other two methods. The 1-2 AC is responsible for a "hump" in both the ground and first excited states, while around the 2-3 AC we observe a clear artifact for the $1^{1} \Sigma^{+}$state and, again, a small hump on the $3^{1} \Sigma^{+}$curve. Remarkably, besides the issues in the AC regions, the MS-CASPT2 PECs fall right on top of the MRCISD ones: this is not the case for a 2-state calculation, in which the three methodologies provide three distinct results. Around the equilibrium distance MSCASPT2 is in very good agreement with MR-

\footnotetext{
${ }^{3}$ Note that the presence of at least one doublyoccupied orbital is important in order to have contributions from all possible excitation classes in a secondorder perturbation theory approach, allowing for a fair comparison with the MRCISD method.
} 


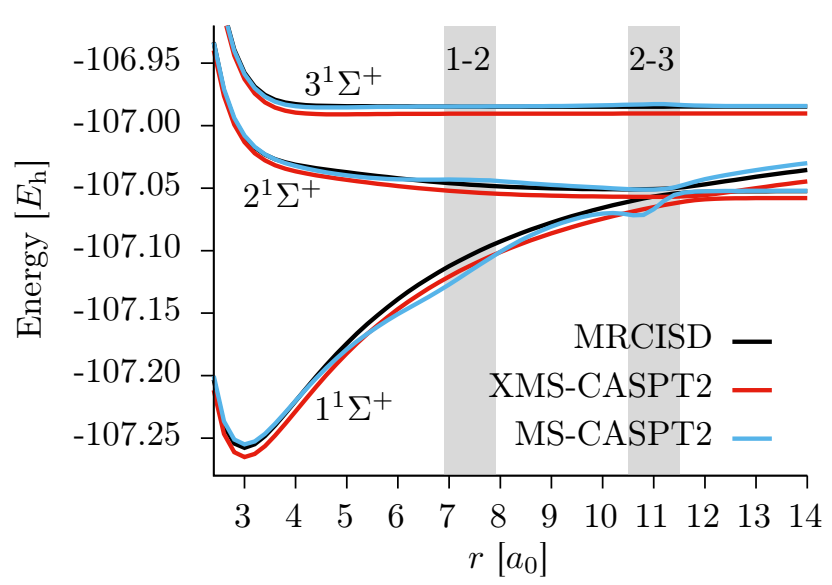

Figure 2: Potential energy curves of the three lowest ${ }^{1} \Sigma^{+}$states of lithium fluoride. The zones highlighted in gray correspond to the avoided crossing regions at the CASSCF level of theory.

CISD, with transition energies to the first and second excited states underestimated by only 0.05 and $0.11 \mathrm{eV}$, respectively. In contrast, XMS-CASPT2 overestimates these excitations by $0.2 \mathrm{eV}$ and $0.25 \mathrm{eV}$, respectively. On the other hand, the plot shown in Figure 2 demonstrates the effectiveness of XMS-CASPT2 in correcting the erratic behavior of the original theory, with PECs that are smooth throughout the entire range of $r$.

Let us now investigate the performance of XDW-CASPT2 and study the dissociation of $\mathrm{LiF}$ as a function of the exponent $\zeta$. Recalling that for $\zeta=0$ all states receive the same weight irrespective of their energy difference (and thus the methodology is exactly equivalent to XMSCASPT2), we show in Figure 3 the results obtained by setting $\zeta=50$. The XDW-CASPT2 potential energy curves substantially overlap the XMS-CASPT2 ones for most of the dissociation, showing no sign of artifacts at any place. Crucially, the $1^{1} \Sigma^{+}$state, and to some extent the $2^{1} \Sigma^{+}$and $3^{1} \Sigma^{+}$states, smoothly slide over to the MS-CASPT2 curves for $r<5 a_{0}$, with an excellent agreement around the equilibrium distance. To rationalize this result, we first analyze the structure of the transformation matrix that diagonalizes $\hat{f}^{s a}$ and inspect the magnitude of zeroth-order mixing among the states. In Figure 4 we show the absolute value of the rotation matrix elements $U_{\beta \alpha}$ (cf. Equation (15)) as

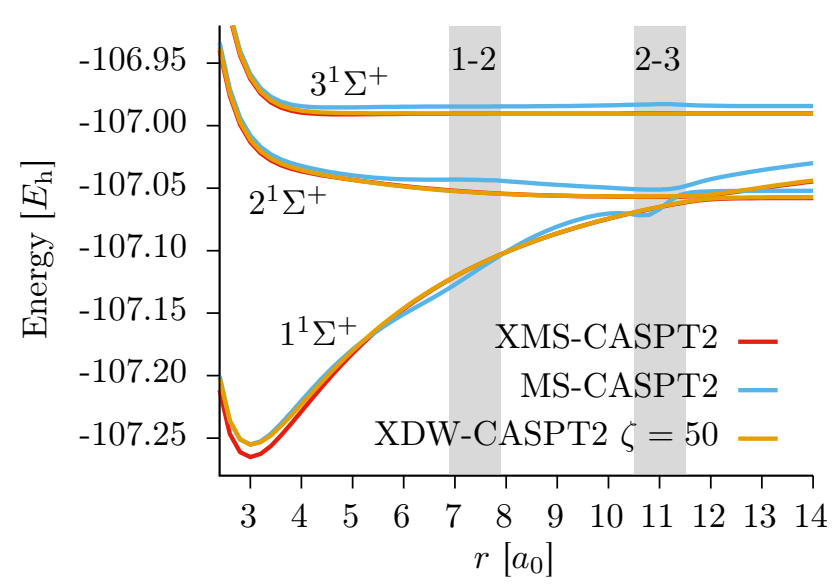

Figure 3: Potential energy curves of the three lowest ${ }^{1} \Sigma^{+}$states of lithium fluoride. Note that to a large extent the XMS-CASPT2 curves are covered by the XDW-CASPT2 ones.

a function of the internuclear distance. The top plot represents the components of the ground state wave function. The magnitude of $U_{31}$, i.e. the curve corresponding to $\beta=3$, never exceeds 0.25 for the entire range of distances, meaning that the contribution of $\Psi_{3}^{(0)}$ to $\tilde{\Psi}_{1}^{(0)}$ is very limited. In contrast, the magnitude of $U_{21}$, i.e. the curve corresponding to $\beta=2$, increases when approaching $r \approx 6.75 a_{0}$, with a peak in the vicinity of the SA-CASSCF avoided crossing. Reciprocally, $U_{11}$ decreases in the same region substantially attaining the same value of $U_{21}$ at $r \approx 6.75 a_{0}$, implying an equal mix of these two states. Lastly, note that $U_{11}$ is approximately 1 for the most part of the plot, that is the off-diagonal elements of $\hat{f}^{s a}$ are very small and therefore $\tilde{\Psi}_{1}^{(0)} \approx \Psi_{1}^{(0)}$. An analogous analysis for the other two plots leads to the following general observations. The magnitude of mixing is a signature of the quasidegeneracies between the states: around $6.75 a_{0}$ the ground and first excited states are equally mixed, while the first and second excited states mix just before $11 a_{0}$. Both cases are around the ACs. At $r=3 a_{0}$, $U_{11} \approx U_{22} \approx U_{33} \approx 1$, meaning that the original CASSCF wave functions are barely coupled by $\hat{f}^{s a}$ and thus remain virtually the same after the transformation.

To further understand the results shown in Figure 3, the weights used in the construction of the density matrices are depicted in Figure 5 


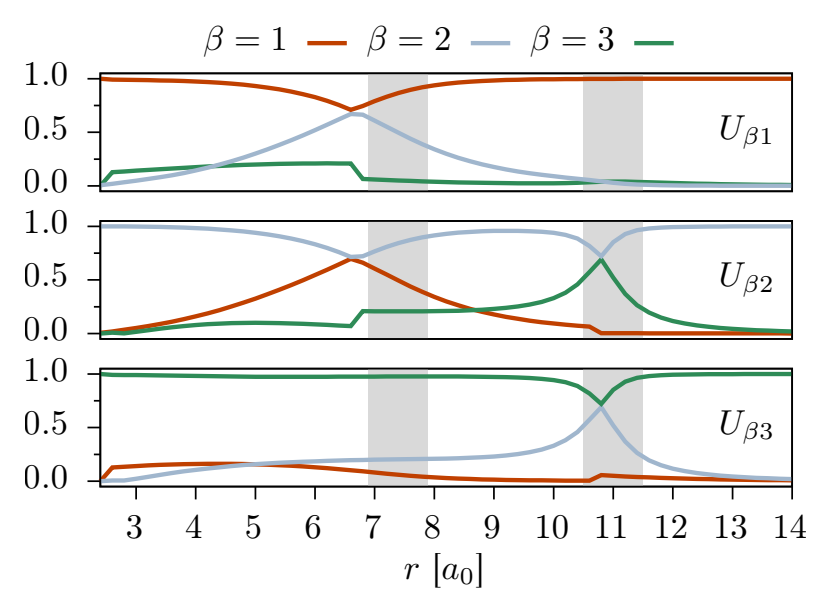

Figure 4: Absolute value of the elements $U_{\beta \alpha}$ of the rotation matrix mixing the zeroth-order CASSCF wave functions. The ground state $(\alpha=1)$ is shown at the top, the first excited state $(\alpha=2)$ in the center and the second excited state $(\alpha=3)$ at the bottom. The zones highlighted in gray correspond to SA-CASSCF ACs.

in a plot similar to the one for $U_{\beta \alpha}$. At $r=3$ $a_{0}$, the ground state weight $\omega_{1}^{\beta}$ with $\beta=1$ is about 0.80 , meaning that $\overline{\mathbf{D}}^{1}$ closely resembles $\mathbf{D}^{1}$, thereby resulting in a Fock operator similar to the MS-CASPT2. The latter is ultimately responsible for the very good agreement between the XDW-CASPT2 and the MS-CASPT2 energy. The densities of the other two states are instead approximately a 50\% mixture (central and bottom plots); as a consequence, the energy of the $2^{1} \Sigma^{+}$and $3^{1} \Sigma^{+}$states is somewhere inbetween the MS-CASPT2 and XMS-CASPT2 one. Note that such straightforward analogies are facilitated by the fact that the zeroth-order states are very weakly coupled through $\hat{f}^{s a}$ at $r=3 a_{0}$. In case of strong mixing, such an analysis would be much harder. At geometries with $r>6 a_{0}$, the weights are roughly equal for all the states. This results in Fock operators $\hat{\bar{f}}^{\alpha}$ resembling $\hat{f} s a$ for $\alpha=1,2,3$ and thus XDW-CASPT2 essentially performs as XMSCASPT2.

The invariance properties of XDW-CASPT2 rely on the assumption made in Equation (19), i.e. that the off-diagonal terms $\bar{f}_{\alpha \beta}^{\gamma}=$ $\left\langle\tilde{\Psi}_{\alpha}^{(0)}\left|\hat{\bar{f}}^{\gamma}\right| \tilde{\Psi}_{\beta}^{(0)}\right\rangle$ are approximately zero. It is

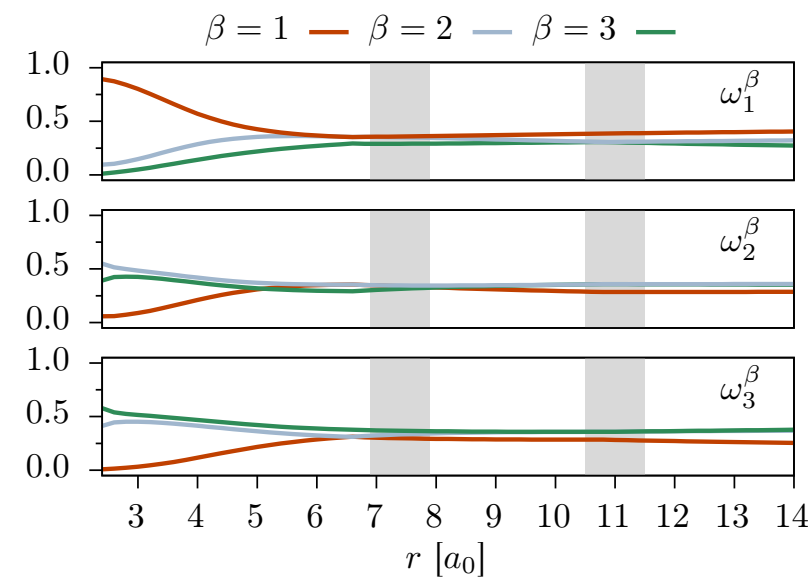

Figure 5: Weights $\omega_{\alpha}^{\beta}$ for $\zeta=50$. The ground state $(\alpha=1)$ is shown at the top, the first excited state $(\alpha=2)$ in the center and the second excited state $(\alpha=3)$ at the bottom.

interesting to investigate if this is the case for LiF. In Figure 6 (a) we show the absolute values of the Fock couplings for each of the three states. The largest elements are observed for the ground state around the equilibrium distance; this is not surprising since the Fock operator is essentially state-specific in that region. On the other hand the opposite is true past $r=6 a_{0}$, with the three Fock operators being roughly equivalent and equal to $\hat{f}^{s a}$ (cf. Figure 5). Recalling that the rotated zerothorder states diagonalize $\hat{f}^{s a}$, their coupling must be approximately zero. The elements $\bar{f}_{\alpha \beta}^{\alpha}$, albeit different from zero, are in practice small enough to yield smooth potential energy curves. As a matter of comparison, the MS-CASPT2 zeroth-order off-diagonal elements between the original CASSCF states are shown in Figure 6 (b): the difference is striking, with values that are one order of magnitude larger compared to XDW-CASPT2. The strongest couplings are around the avoided crossings, exactly where MS-CASPT2 performs poorly.

Increasing the value of $\zeta$ sharpens the transition between state-specific and state-average regimes. As already observed in the context of DW-DSRG, ${ }^{26}$ this leads to the appearance of wiggles along the potential energy curves due to sudden changes of the zeroth-order weights. This behavior can be seen in Figure 7 for $\zeta=$ 5000. For instance, near the 1-2 AC, the XDW- 
CASPT2 curve for state $2^{1} \Sigma^{+}$rapidly switches between the XMS-CASPT2 and MS-CASPT2 references. Inspection of the weights in Fig-
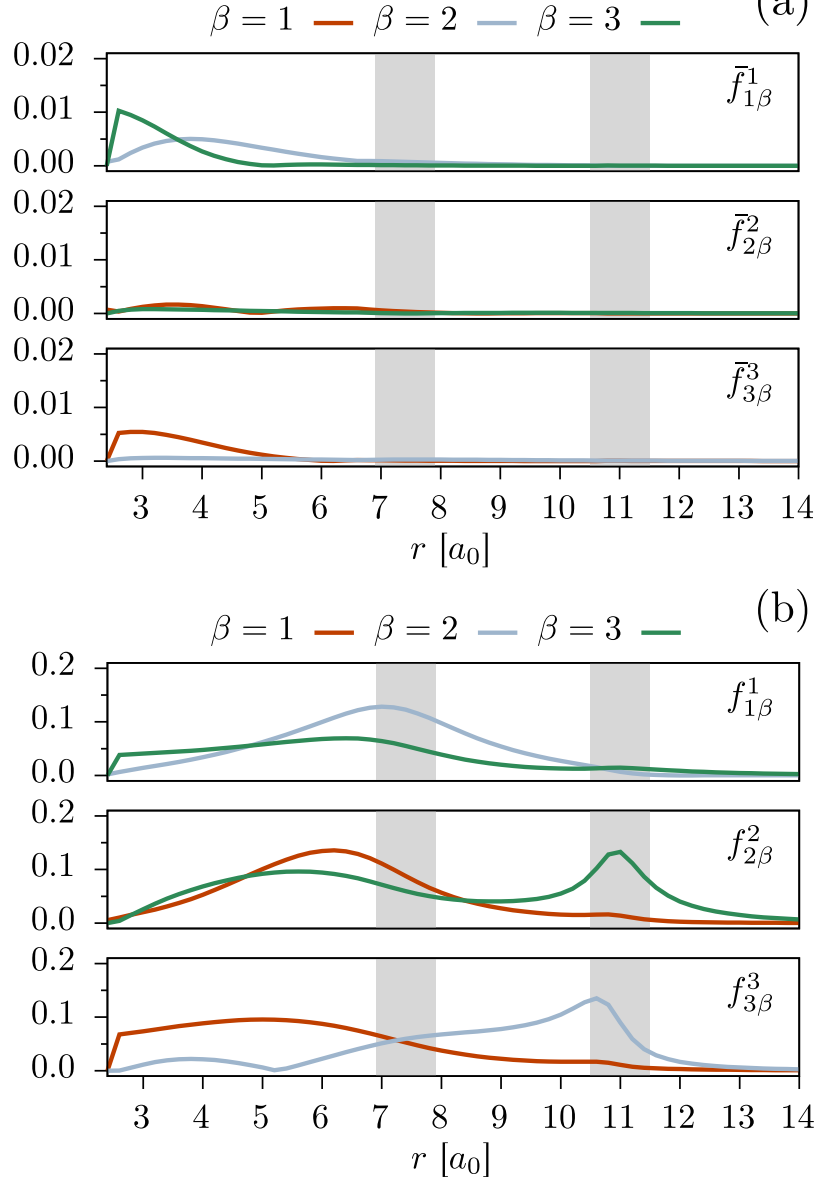

Figure 6: Absolute values of the Fock operator off-diagonal entries for (a) XDW-CASPT2 with $\zeta=50$ (elements $\bar{f}_{\alpha \beta}^{\gamma}$ ) and (b) MS-CASPT2 (elements $f_{\alpha \beta}^{\gamma}$ ). For each method (three plots), the ground state $(\alpha=1)$ is shown at the top, the first excited state $(\alpha=2)$ in the center and the second excited state $(\alpha=3)$ at the bottom. Note that the Fock operator used to compute the couplings is different for each state and only the case $\gamma=\alpha$ is of relevance.

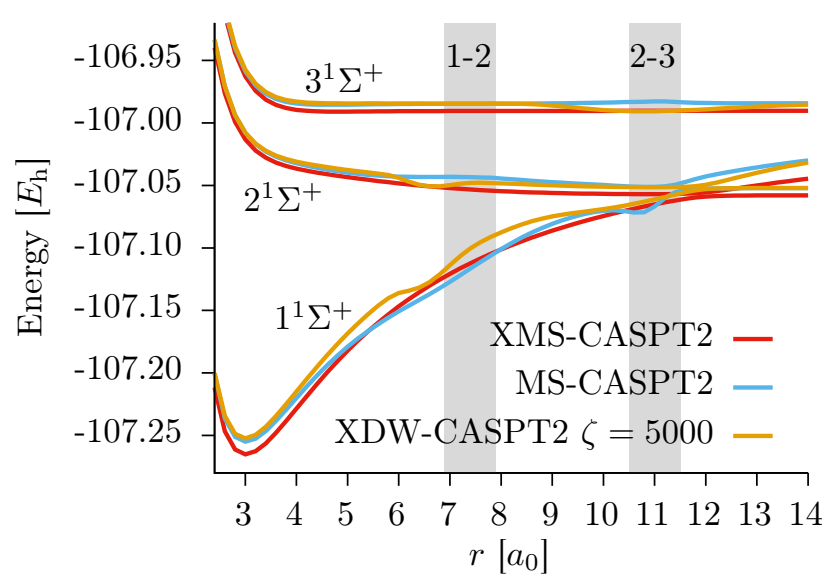

Figure 7: Potential energy curves of the three lowest ${ }^{1} \Sigma^{+}$states of lithium fluoride.

ure 8 reveals a clear correlation between the weights $\omega_{\alpha}^{\beta}$ and these oscillations. Whenever the weights undergo a rapid and significant change, the energy does so accordingly. De-

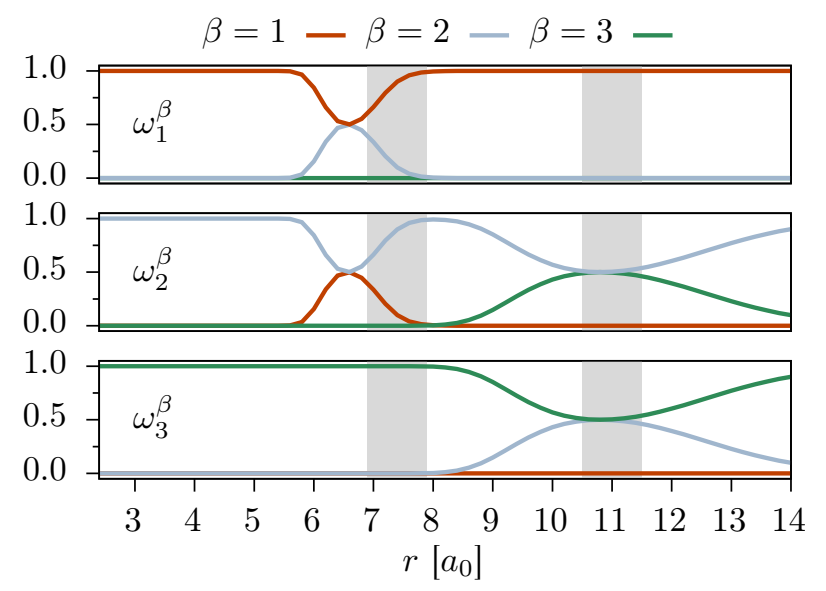

Figure 8: Weights $\omega_{\alpha}^{\beta}$ for $\zeta=5000$.

spite this oscillatory behavior, the off-diagonal elements of the Fock operator for $\zeta=5000$ are in the same order of magnitude as for $\zeta=50$, hence still 10-fold less than MS-CASPT2, as can be seen in Figure 9. Therefore, it appears that the cause of the wiggles in the PECs is not due to the diagonal approximation.

Lastly, the results obtained taking the limit $\zeta \rightarrow \infty$ are reported in Figure 10. For this case the weights never change and correspond to unit vectors, hence the densities 


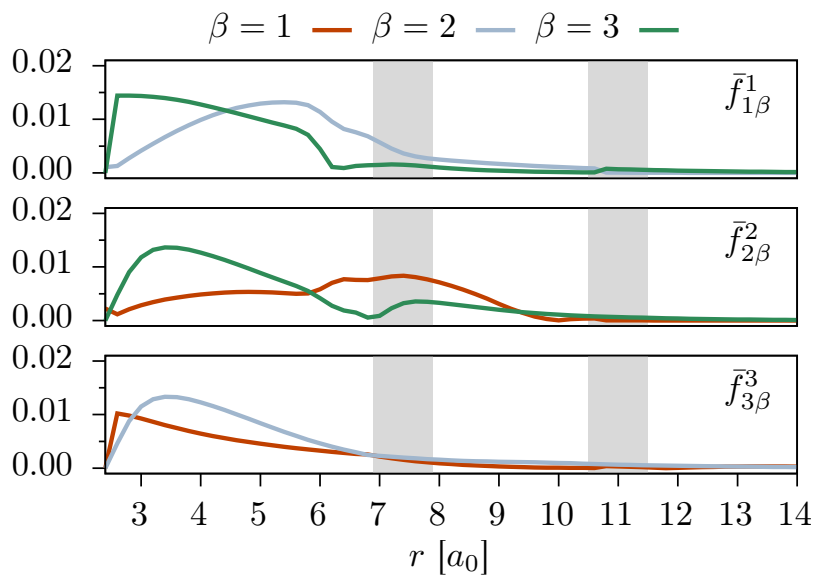

Figure 9: Absolute values of the elements $\bar{f}_{\alpha \beta}^{\alpha}$ for $\zeta=5000$.

are state-specific: $\overline{\mathbf{D}}^{\alpha}=\tilde{\mathbf{D}}^{\alpha}$. This leads to potential energy curves that mostly overlap with the MS-CASPT2 ones, however without the artifacts around the SA-CASSCF neardegeneracies. Notably, state-specific Fock oper-

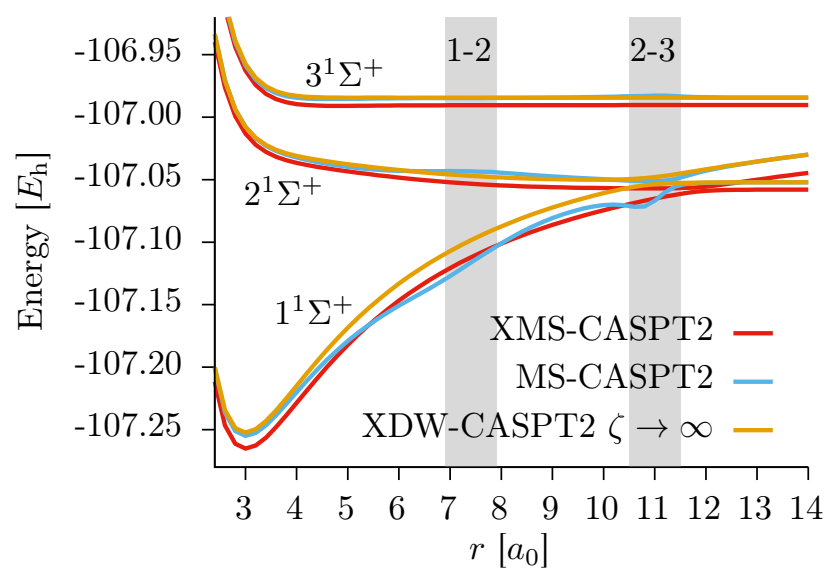

Figure 10: Potential energy curves of the three lowest ${ }^{1} \Sigma^{+}$states of lithium fluoride.

ators built with densities $\tilde{\mathbf{D}}^{\alpha}$ do not couple the states as strong as the original operators, $\hat{f}^{\alpha}$, since the zeroth-order off-diagonal elements for $\zeta \rightarrow \infty$ are as large as those for $\zeta=5000$ (see Supporting Information). This result is important because it corroborates the conjecture that the PEC wiggles observed for intermediate values of $\zeta$ are strictly caused by the rapid change of the weights.

\subsection{Conical intersection in allene}

Projection of the zeroth-order Hamiltonian onto the individual states of the model space defines a MRPT that is not invariant under unitary transformation of the model states. Failure to satisfy Equation (19) leads to unphysical results at conical intersections or in the vicinity of avoided crossings. This situation has been already observed in the LiF dissociation, however a more challenging test is that of the minimum energy conical intersection (MECI) of the distorted allene molecule, depicted in Figure 11. Around that point, the $1^{1} \mathrm{~A}^{\prime}$ and $2^{1} \mathrm{~A}^{\prime}$ states

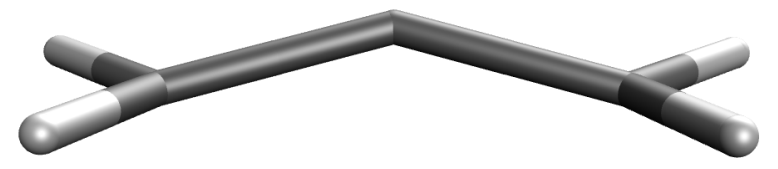

Figure 11: The $1^{1} \mathrm{~A}^{\prime}$ and $2^{1} \mathrm{~A}^{\prime}$ MECI geometry of the allene molecule.

are quasidegenerate and thus only the space spanned by them is well-defined. In order to investigate the behavior of the various CASPT2 variants in this situation, we performed twodimensional, non-relaxed scans by varying the $\mathrm{C}-\mathrm{C}-\mathrm{C}$ bend angle and the $\mathrm{C}-\mathrm{C}-\mathrm{C}-\mathrm{H}$ torsional angle in steps of 0.25 degrees in the range of -10 to +10 degrees from the CASSCF MECI point, respectively ${ }^{4}$. The computational details are the same as in Ref. 23, and are fully described in the Supporting Information; here we report only the essential points. The reference wave functions were obtained in a SA-CASSCF calculation with 4 electrons in 3 orbitals of $\mathrm{a}^{\prime}$ symmetry and 1 orbital of $a^{\prime \prime}$ symmetry. This amounts to a complete active space of 12 totally symmetric configuration state functions, thus allowing to study the behavior of the potential energy surface as a function of the number of states, up to the complete active space limit. Given that the calculation focuses on the $1^{1} \mathrm{~A}^{\prime}$ and $2^{1} \mathrm{~A}^{\prime}$ conical intersection, the CASSCF orbital optimization was carried out for the two

\footnotetext{
${ }^{4}$ Note that the $\mathrm{C}-\mathrm{C}-\mathrm{C}-\mathrm{H}$ torsion angle is simultaneously changed on both sides of the molecule in order to preserve the $\mathrm{C}_{s}$ symmetry.
} 
lowest states only, while the remaining 10 states were obtained by diagonalization of the configuration interaction matrix. The basis set used was the GAMESS (Us)-style variation of the Dunning-Hays basis, augmented by a single polarization spherical $d$ function on each carbon.

In Figure 12 we report the colormapped isosurface plots of the energy difference between the $1^{1} \mathrm{~A}^{\prime}$ and $2^{1} \mathrm{~A}^{\prime}$ states computed with a model space spanned by the 2 lowest roots only. Figure 12 (a) shows the result obtained with MSCASPT2. At the CASSCF MECI point the origin of the plot - there is a singularity and the surface around this point is completely compromised, showing the deficiency of this methodology. On the contrary, as can be seen from Figure 12 (b), the surface obtained with XMS-CASPT2 does not show any sign of artifacts, demonstrating the importance of invariance to obtain physically sound results. The plot in Figure 12 (c) illustrates the behavior of XDW-CASPT2 for $\zeta=50$. The surface is virtually identical to that obtained with XMSCASPT2, a result that is easily explained upon analyzing the density weights. Recalling that the model space only has a dimension of two, the largest and smallest values of $\omega_{\alpha}^{\beta}$ observed in the entire scan were 0.53 and 0.47 , respectively, meaning that the difference between the XDW-CASPT2 and XMS-CASPT2 partitions are very small across the board. Lastly, in Figure $12(\mathrm{~d})$ is shown the surface obtained by letting $\zeta \rightarrow \infty$. Remarkably, albeit the use of purely state-specific operators, the PES around the MECI point is perfectly smooth. The overall morphology is analogous to the last two cases, even though a slightly larger width of the potential well is noticeable.

Repeating the same calculation with XMSCASPT2 including all 12 states of the complete active space results again in a smooth surface as shown in Figure 13 (a). The position of the MECI at the correlated level changes according to the number of states, and for XMS-CASPT2 is substantially converged with a model space of 6 states (see Supporting Information). In Figure 13 (b) and (c) we report the result obtained with XDW-CASPT2 for $\zeta=50$ and $\zeta \rightarrow \infty$.
Once again the PESs are smooth everywhere and on par with the XMS-CASPT2 one. Moreover, we note that all three plots of Figure 13 are remarkably similar to the one obtained by Granovsky ${ }^{23}$ with extended MCQDPT. In contrast to the 2-state case, the dynamical weights obtained with $\zeta=50$ are significantly different from the state-average ones. The Fock operators $\hat{\bar{f}}^{1}$ and $\hat{\bar{f}}^{2}$ are substantially defined by the first four states, since the weights assigned starting from the fifth one are less than 0.03 , thereby contributing little to nothing to the $1^{1} \mathrm{~A}^{\prime}$ and $2^{1} \mathrm{~A}^{\prime} 1$-RDMs. Crucially, this does not imply that PESs obtained with a model space of dimension four are the same as those obtained with one of higher dimension. Both Equation (15) and Equation (23) directly depend on the total number of model states and their wave function. As a result, different model space dimensions give rise to distinct partitionings of $\hat{H}$, which are ultimately coupled together in a non-trivial way through the formation of the second-order effective Hamiltonian.

\subsection{Vertical excitation energies}

One of the design objectives of XDW-CASPT2 is to maintain the accuracy of MS-CASPT2 in the calculation of transition energies. If we consider a molecule in its electronic ground state, the dynamical weighting scheme is such that when the energy gap to the first excited state is larger than $\zeta^{-1 / 2}$, then the density matrices of both these states will barely mix with each other, remaining predominantly state-specific. It is reasonable to assume that in this situation the energy separation between these states is sizable and that their associated wave functions have well-defined, but distinct character. Hence, the rotated reference states obtained from Equation (15) will be similar to the original ones: $\tilde{\Psi}_{\alpha}^{(0)} \approx \Psi_{\alpha}^{(0)}$. Under these circumstances, all the quantities in XDW-CASPT2 will not be very different from those in MSCASPT2, such that we expect the two methods to have a comparable accuracy. This was indeed observed in the previous section for lithium fluoride. In principle, the same logic applies when the model space dimension is larger than two: 


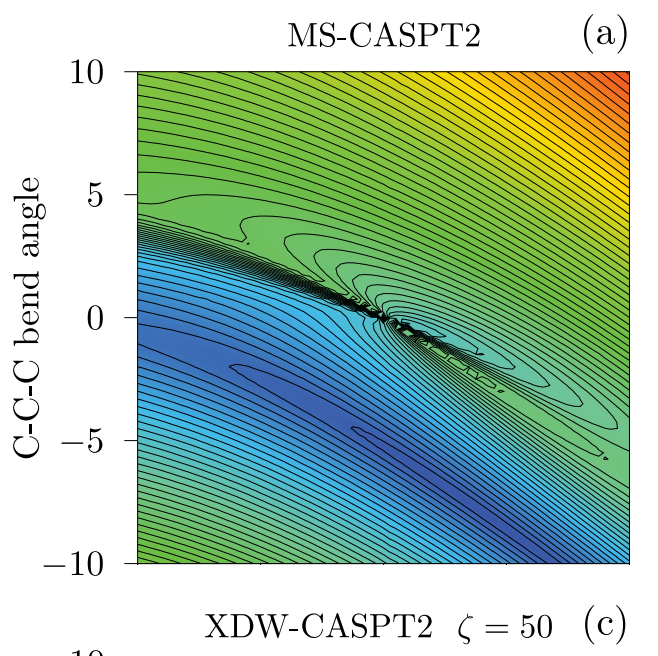

(b) XMS-CASPT2
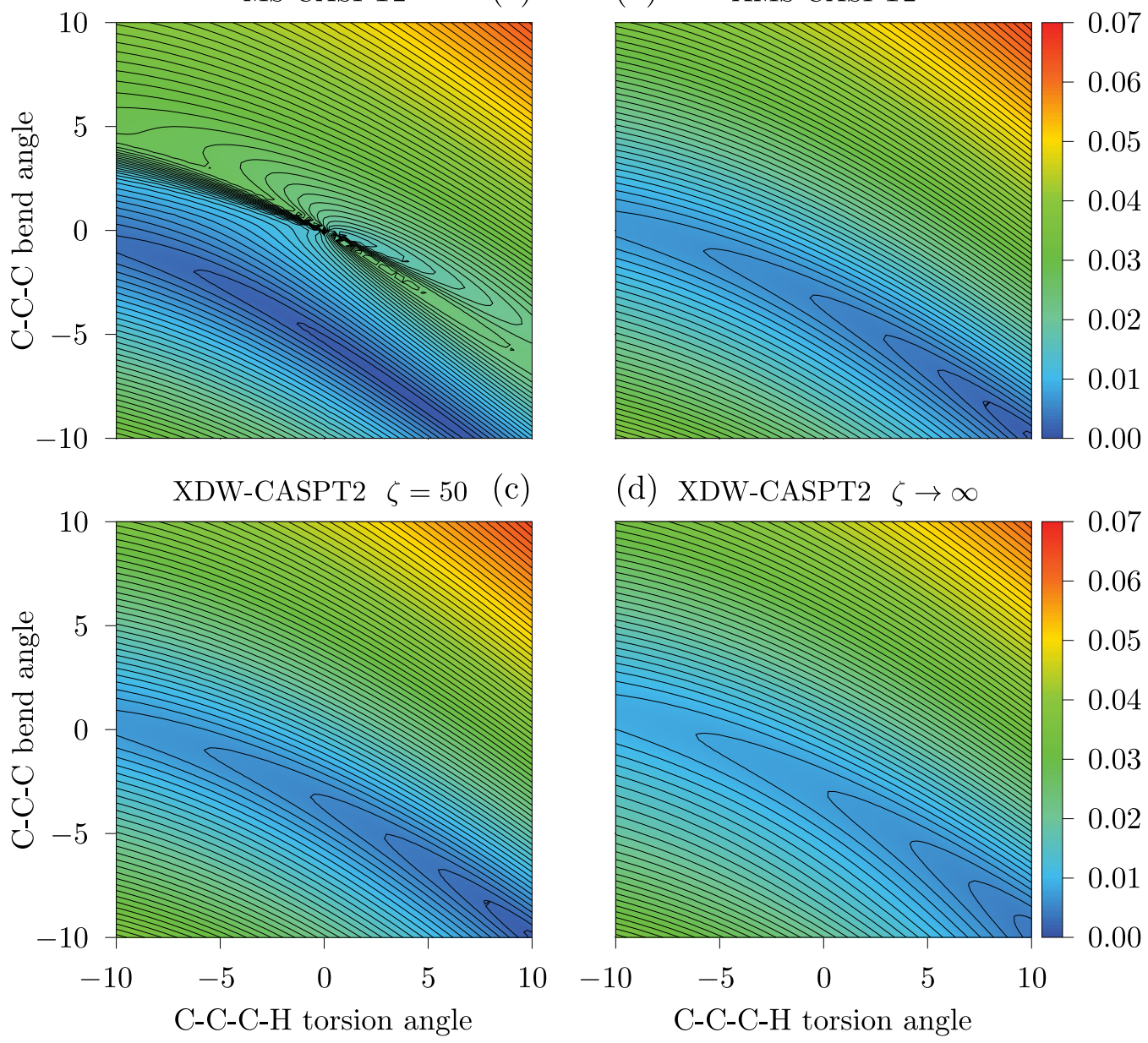

(d) XDW-CASPT2 $\zeta \rightarrow \infty$

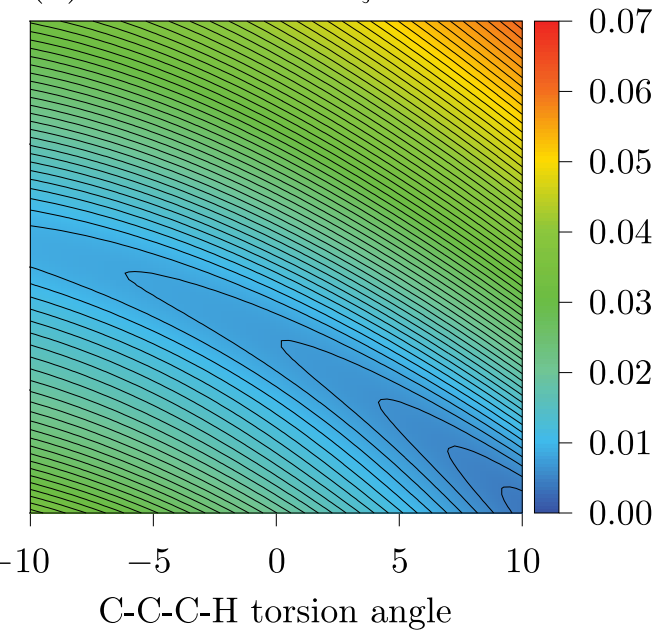

Figure 12: Colormapped isosurface plot of the absolute energy difference (in $E_{\mathrm{h}}$ ) between the $1^{1} \mathrm{~A}^{\prime}$ and $2^{1} \mathrm{~A}^{\prime}$ states for a model space including 2 states. The same calculation was carried out with different methodologies: (a) MS-CASPT2, (b) XMS-CASPT2, (c) XDW-CASPT2 with $\zeta=50$ and (d) XDW-CASPT2 with $\zeta \rightarrow \infty$. 

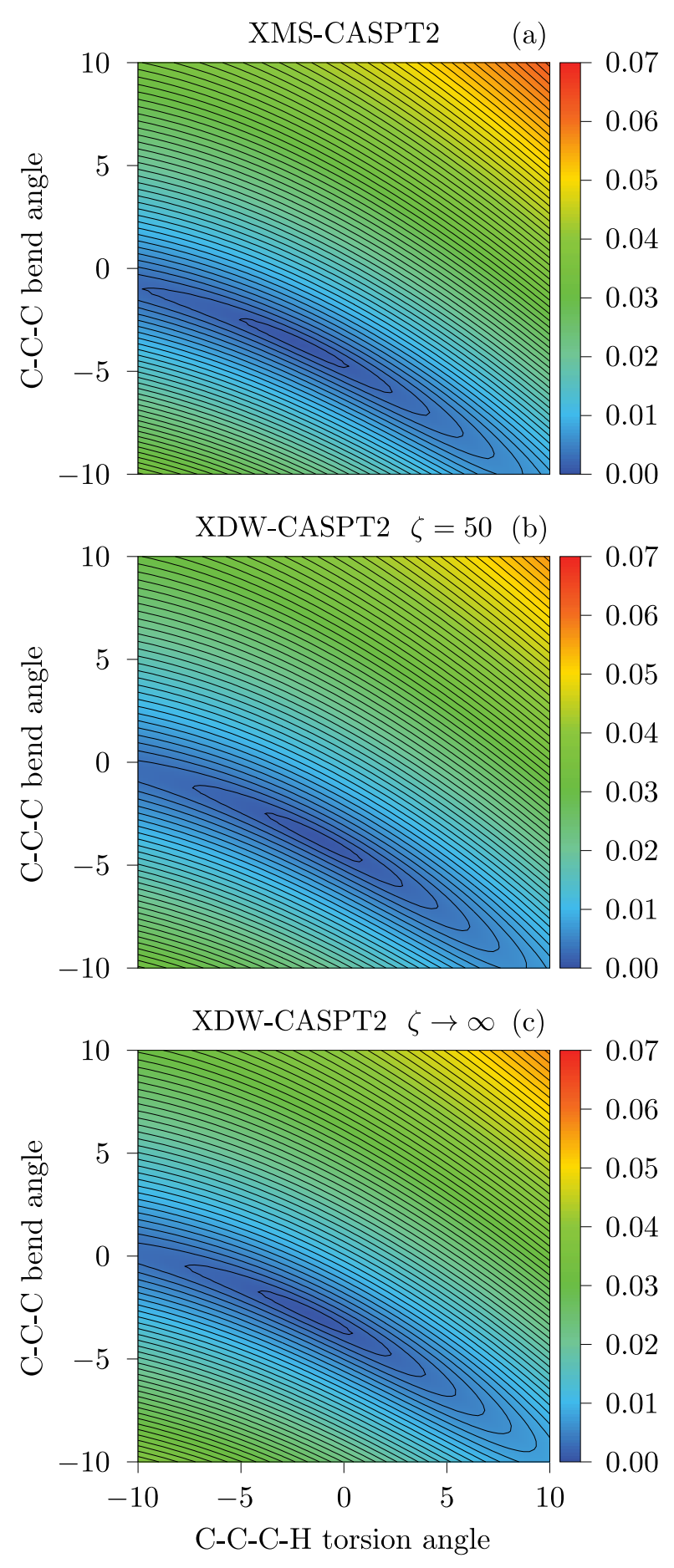

Figure 13: Colormapped isosurface plot of the absolute energy difference between the $1^{1} \mathrm{~A}^{\prime}$ and $2^{1} \mathrm{~A}^{\prime}$ states for a 12 -state model space computed with different methodologies: (a) XMSCASPT2, (c) XDW-CASPT2 $(\zeta=50)$ and (c) $\mathrm{XDW}-\mathrm{CASPT} 2(\zeta \rightarrow \infty)$. as long as all states are energetically well separated from each other and the rotated reference wave functions maintain their original character, we expect similar results for XDWCASPT2 and MS-CASPT2. A different and much more complicated situation occurs when many model states lie within a limited region of the spectrum and interact strongly with each other at zeroth-order. Although it is conceptually easy to visualize the amount of density mixing by inspecting the weights $\omega_{\alpha}^{\beta}$, the fact that the rotated model states are linear combinations of the original reference wave functions, makes it hard to rationalize the physical content of the Fock operator in these terms. The three different cases are summarized in Scheme 1.

In order to assess the accuracy of XDWCASPT2 for the calculation of electronically excited states, we computed the vertical energy gap between the ground and the first excited singlet state for a series of small to medium organic compounds and compared the results to MS-CASPT2. This case corresponds to the first scenario illustrated in Scheme 1. The molecules were taken from Thiel's benchmark set, ${ }^{21}$ excluding ethene and cyclopropene since no singlet excited state was considered for these two systems. In order to appreciate the effects of the dynamical weighting scheme, the calculated first excited states always belonged to the same irreducible representation as the ground state. The geometries were taken from Ref. 21 and correspond to structures optimized at MP2/6$31 \mathrm{G}^{*}$ level of theory. The reference wave functions were obtained by a 2-state SA-CASSCF calculation using the TZVP basis set ${ }^{38}$ and the RICD approximation. ${ }^{39}$ Full computational details are available in the Supporting Information. Vertical transition energies were calculated with MS-CASPT2, XMS-CASPT2 and XDW-CASPT2 with two values of $\zeta$ and setting the IPEA shift to zero for all methods. A real shift was used when necessary and equally applied to all methods in order to obtain comparable energies.

In Figure 14 is reported the deviation of XMSCASPT2 and XDW-CASPT2 vertical excitation energies with respect to MS-CASPT2, which is used as the reference since the de- 


\section{case I}

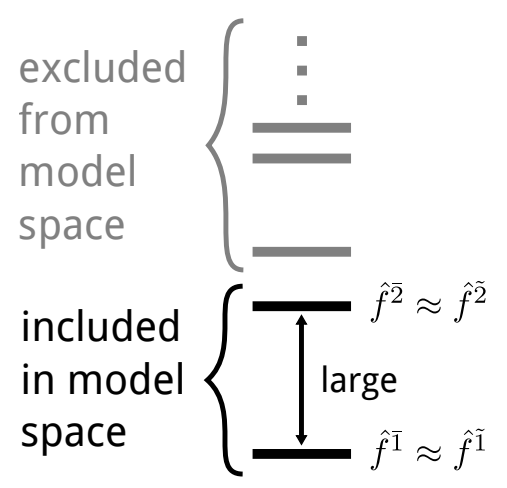

case II

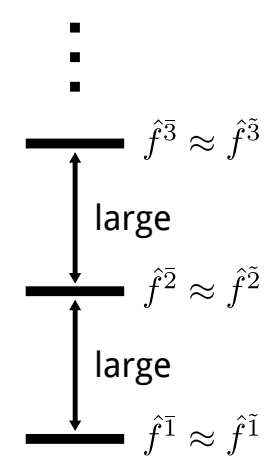

case III

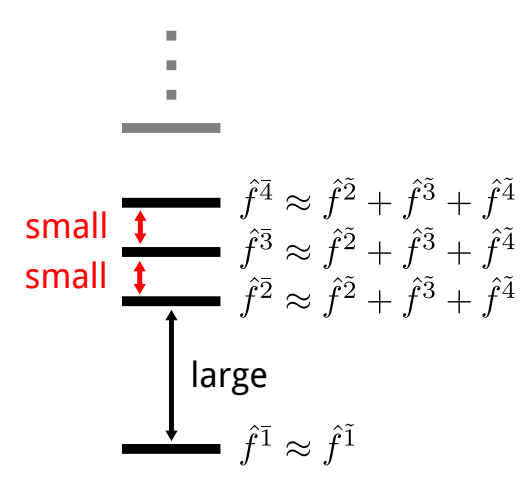

Scheme 1: Three main scenarios for the calculation of excited states energies. In case I only the well-separated ground and first excited states are included in the model space. In case II many states are included in the calculation, but all of them are well separated. In case III several lowlying excited states are included in the model space and these are energetically very close to each other. Therefore, their Fock operators will be approximately state-average in contrast to the other cases.

sign objective is to reproduce its value. The largest deviation is observed for XMS-CASPT2, with a general tendency to slightly overestimate the excitation energies by up to $0.2 \mathrm{eV}$. In two cases, acetone and formaldehyde, the energy is instead considerably underestimated: these transitions correspond to the largest of the entire set, $8.93 \mathrm{eV}$ and $10.06 \mathrm{eV}$, respectively, and highlight the difficulty of the stateaverage Fock operator to deal with states that energetically so far apart. Note that the MSCASPT2 values, $9.28 \mathrm{eV}$ and $10.40 \mathrm{eV}$, agree well with CC3/TZVP ${ }^{21}$ with transitions estimated at $9.65 \mathrm{eV}$ and $10.45 \mathrm{eV}$, such that the underestimation of XMS-CASPT2 appears to be a true deficiency of the methodology. The results of XDW-CASPT2 are instead on par with MS-CASPT2. The values for the transitions are exactly reproduced for several systems, more so for $\zeta \rightarrow \infty$ than $\zeta=50$, even though the general performance of both is virtually the same. The accuracy of the three methodologies is well captured by normal distributions of the energy deviations with respect to MS-CASPT2 as shown in Figure 15. Note that we do not want to make any claim that the deviations are normally distributed, but just point out that this plot neatly summarizes the results shown in Figure 14. Despite the mean of all three methods is quite close to the MSCASPT2 one, XDW-CASPT2 is clearly a more reliable choice than XMS-CASPT2, at least for the scenario considered here. In terms of mean absolute deviations, XMS-CASPT2 excitation energies differ by $0.12 \mathrm{eV}$ on average, whereas the agreement is excellent for XDW-CASPT2, with a discrepancy of only $0.02 \mathrm{eV}$ and $0.01 \mathrm{eV}$ for $\zeta=50$ and $\zeta \rightarrow \infty$, respectively.

\section{Conclusions}

In this work we have proposed and investigated a new variant of the CASPT2 method. By a careful analysis of the properties of MSCASPT2 and XMS-CASPT2, we have identified the two key components that characterize the success of each variant and included them in the newly developed XDW-CASPT2 approach. First, diagonalization of the state-average Fock operator in the reference basis provides a new set of zeroth-order states. Second, this is followed by the construction of state-specific Fock operators with dynamically adjusted weights that depend on the energy separation between the states. These operators are then used to partition the Hamiltonian in a MS-CASPT2 calculation. The resulting method is approximately invariant under unitary transforma- 


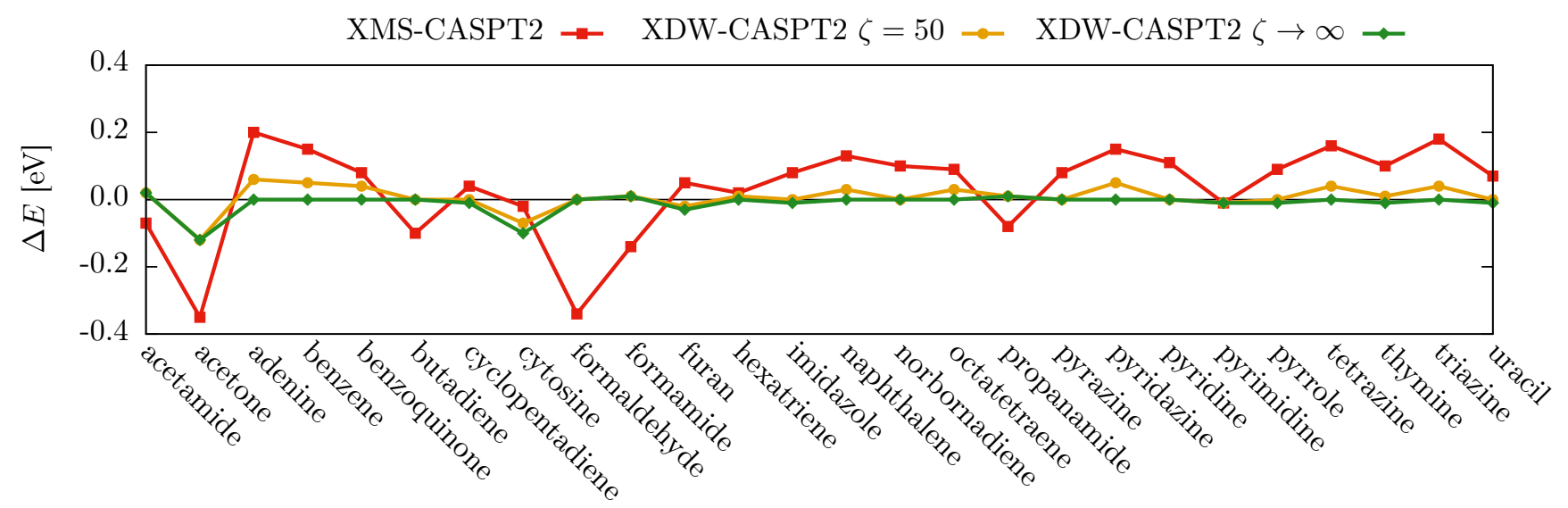

Figure 14: Signed deviations of singlet vertical excitation energies with respect to MS-CASPT2.

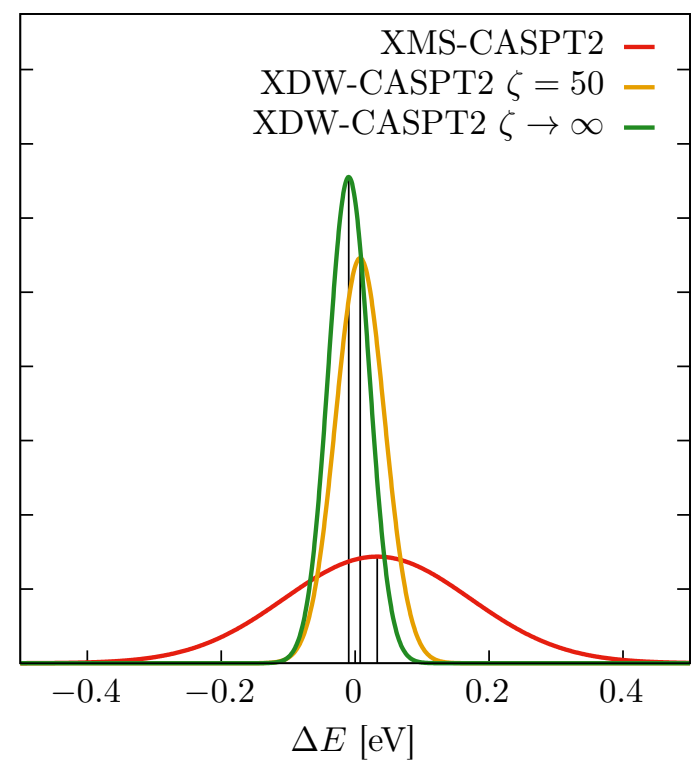

Figure 15: Normal distributions of excitation energy deviations with respect to MS-CASPT2. tions of the model states, a property that ensures a physical behavior in the vicinity of avoided crossings and conical intersections, and at the same time shows an accuracy comparable to conventional MS-CASPT2. The dynamical weighting scheme introduces a parameter $\zeta$ which acts as a threshold controlling the statespecificity of the Fock operator, thereby allowing the method to interpolate between XMSCASPT2 and MS-CASPT2 (with rotated reference functions). Importantly, even though XDW-CASPT2 employs the diagonal approximation, in practice it approximately satisfies all important properties listed by Granovsky ${ }^{23}$.

The reliability of XDW-CASPT2 is demonstrated in the typical benchmark system $\mathrm{LiF}$, whose avoided crossings represent a difficult task for multireference approaches. The obtained potential energy curves overlap with the XMS-CASPT2 ones in the regions where the underlying zeroth-order states are quasidegenerate, hence do not show the wiggles typical of MS-CASPT2, but at the same time the vertical transitions to the first two excited states are in better agreement than XMS-CASPT2 with the reference MRCISD values. The robustness of XDW-CASPT2 is further tested by studying the conical intersection in the allene molecule, for which smooth PESs where obtained for different values of $\zeta$ and dimensions of the model space. At last, vertical excitation energies are shown to be in almost perfect agreement with MS-CASPT2 for singlet transitions in a set of 26 organic compounds, unlike XMS-CASPT2 
that shows an average deviation in the order of $0.1 \mathrm{eV}$ and maximum deviations as large as 0.4 $\mathrm{eV}$.

The XDW-CASPT2 method can be viewed as a bridge between MS-CASPT2 and XMSCASPT2, drawing the best from both these approaches and providing a valid alternative to other quasidegenerate multireference perturbation theories. Moreover, being based on the CASPT formalism constitutes a practical advantage: any existing implementation can be easily adapted to provide XDW-CASPT2 as an option and at the same time it only requires an additional input parameter from the final user. The similarity with its parent theory also means that XDW-CASPT2 can also be used with zeroth-order wave functions obtained with modern approaches such as the density matrix renormalization group. ${ }^{40}$ From the computational perspective, the only difference with (X)MS-CASPT2 is a small overhead for the construction of the dynamically weighted densities and thus it is applicable to the same kind of systems where the parent methods are an option. At last, we envision XDW-CASPT2 to be a very interesting method in the context of $a b$ initio molecular dynamics, once the restriction of imposing molecular symmetries is lifted, e.g. through the use of the off-diagonal elements of the full Hamiltonian.

Acknowledgement S.B. acknowledges the Swiss National Science Foundation (SNSF) for the funding received through the Early Postdoc.Mobility fellowship (grant number P2SKP2_184034). R.L. acknowledges the Swedish Research Council (VR, grant number 2016-03398).

\section{Supporting Information Avail- able}

The following files are available free of charge. In the Supporting Information we provide additional results on the dissociation of lithium fluoride and on the conical intersection of the distorted allene molecule and we report supplementary computational details on the allene molecule as well as information necessary to reproduce the calculation of the singlet vertical excitation energies.

\section{References}

(1) Matsika, S.; Krylov, A. I. Introduction: Theoretical Modeling of Excited State Processes. Chemical Reviews 2018, 118, 6925-6926.

(2) Vacher, M.; Fdez. Galván, I.; Ding, B.W.; Schramm, S.; Berraud-Pache, R.; Naumov, P.; Ferré, N.; Liu, Y.-J.; Navizet, I.; Roca-Sanjuán, D.; Baader, W. J.; Lindh, R. Chemi- and Bioluminescence of Cyclic Peroxides. Chemical Reviews 2018, 118, 6927-6974.

(3) Norman, P.; Dreuw, A. Simulating Xray Spectroscopies and Calculating CoreExcited States of Molecules. Chemical Reviews 2018, 118, 7208-7248.

(4) Casanova, D. Theoretical Modeling of Singlet Fission. Chemical Reviews 2018, 118, 7164-7207.

(5) Lischka, H.; Nachtigallová, D.; Aquino, A. J.; Szalay, P. G.; Plasser, F.; MacHado, F. B.; Barbatti, M. Multireference Approaches for Excited States of Molecules. Chemical Reviews 2018, 118, 7293-7361.

(6) Cave, R. J.; Davidson, E. R. Quasidegenerate variational perturbation theory and the calculation of firstorder properties from variational perturbation theory wave functions. The Journal of Chemical Physics 1988, 89, 6798-6814.

(7) Nakano, H. Quasidegenerate perturbation theory with multiconfigurational selfconsistentfield reference functions. The Journal of Chemical Physics 1993, 99, 79837992.

(8) Malrieu, J.-P.; Heully, J.-L.; Zaitsevskii, A. Multiconfigurational secondorder perturbative methods: Overview 
and comparison of basic properties. Theoretica Chimica Acta 1995, 90, 167-187.

(9) Hoffmann, M. R. Third-order complete active space self-consistent field based generalized Van Vleck perturbation theory. Chemical Physics Letters 1993, 210, 193200.

(10) Hoffmann, M. R. Canonical Van Vleck Quasidegenerate Perturbation Theory with Trigonometric Variables. The Journal of Physical Chemistry 1996, 100, 6125-6130.

(11) Finley, J.; Malmqvist, P.-Å.; Roos, B. O.; Serrano-Andrés, L. The multi-state CASPT2 method. Chemical Physics Letters 1998, 288, 299-306.

(12) Mahapatra, U. S.; Datta, B.; Mukherjee, D. Development of a size-consistent state-specific multireference perturbation theory with relaxed model-space coefficients. Chemical Physics Letters 1999, 299, 42-50.

(13) Shavitt, I. Multi-state Multireference RayleighSchrödinger Perturbation Theory for Mixed Electronic States: Second and Third Order. International Journal of Molecular Sciences 2002, 3, 639-655.

(14) Angeli, C.; Borini, S.; Cestari, M.; Cimiraglia, R. A quasidegenerate formulation of the second order $n$-electron valence state perturbation theory approach. The Journal of Chemical Physics 2004, 121, 4043-4049.

(15) Fink, R. F. The multi-reference retaining the excitation degree perturbation theory: A size-consistent, unitary invariant, and rapidly convergent wavefunction based ab initio approach. Chemical Physics 2009, 356, 39-46.

(16) Roskop, L.; Gordon, M. S. Quasidegenerate second-order perturbation theory for occupation restricted multiple active space self-consistent field reference functions. The Journal of Chemical Physics 2011, 135, 044101.

(17) Sharma, S.; Jeanmairet, G.; Alavi, A. Quasi-degenerate perturbation theory using matrix product states. The Journal of Chemical Physics 2016, 144, 034103.

(18) Giner, E.; Angeli, C.; Garniron, Y.; Scemama, A.; Malrieu, J.-P. A JeziorskiMonkhorst fully uncontracted multireference perturbative treatment. I. Principles, second-order versions, and tests on ground state potential energy curves. The Journal of Chemical Physics 2017, 146, 224108.

(19) Garniron, Y.; Scemama, A.; Giner, E.; Caffarel, M.; Loos, P.-F. Selected configuration interaction dressed by perturbation. The Journal of Chemical Physics 2018, 149, 064103.

(20) Bozkaya, U. Efficient Implementation of the Second-Order Quasidegenerate Perturbation Theory with Density-Fitting and Cholesky Decomposition Approximations: Is It Possible To Use HartreeFock Orbitals for a Multiconfigurational Perturbation Theory? Journal of Chemical Theory and Computation 2019, 15, 44154429.

(21) Schreiber, M.; Silva-Junior, M. R.; Sauer, S. P. A.; Thiel, W. Benchmarks for electronically excited states: CASPT2, CC2, CCSD, and CC3. The Journal of Chemical Physics 2008, 128, 134110.

(22) Silva-Junior, M. R.; Schreiber, M.; Sauer, S. P. A.; Thiel, W. Benchmarks of electronically excited states: Basis set effects on CASPT2 results. The Journal of Chemical Physics 2010, 133, 174318.

(23) Granovsky, A. A. Extended multiconfiguration quasi-degenerate perturbation theory: The new approach to multi-state multi-reference perturbation theory. The Journal of Chemical Physics 2011, 134, 214113. 
(24) Shiozaki, T.; Gyrffy, W.; Celani, P.; Werner, H.-J. Communication: Extended multi-state complete active space secondorder perturbation theory: Energy and nuclear gradients. The Journal of Chemical Physics 2011, 135, 081106.

(25) Park, J. W. Single-State Single-Reference and Multistate Multireference ZerothOrder Hamiltonians in MS-CASPT2 and Conical Intersections. Journal of Chemical Theory and Computation 2019, 15, 39603973.

(26) Li, C.; Lindh, R.; Evangelista, F. A. Dynamically weighted multireference perturbation theory: Combining the advantages of multi-state and state-averaged methods. The Journal of Chemical Physics 2019, 150, 144107.

(27) Deskevich, M. P.; Nesbitt, D. J.; Werner, H.-J. Dynamically weighted multiconfiguration self-consistent field: Multistate calculations for $\mathrm{F}+\mathrm{H}_{2} \mathrm{O} \rightarrow \mathrm{HF}+\mathrm{OH}$ reaction paths. The Journal of Chemical Physics 2004, 120, 7281-7289.

(28) Glover, W. J. Communication: Smoothing out excited-state dynamics: Analytical gradients for dynamically weighted complete active space self-consistent field. The Journal of Chemical Physics 2014, 141, 171102.

(29) Bloch, C. Sur la théorie des perturbations des états liés. Nuclear Physics 1958, 6, 329-347.

(30) Lindgren, I. The Rayleigh-Schrödinger perturbation and the linked-diagram theorem for a multi-configurational model space. Journal of Physics B: Atomic and Molecular Physics 1974, \%, 2441-2470.

(31) Zaitsevskii, A.; Malrieu, J.-P. Multipartitioning quasidegenerate perturbation theory. A new approach to multireference Møller-Plesset perturbation theory. Chemical Physics Letters 1995, 233, 597604.
(32) Kats, D.; Werner, H.-J. Multi-state local complete active space second-order perturbation theory using pair natural orbitals (PNO-MS-CASPT2). The Journal of Chemical Physics 2019, 150, 214107.

(33) Fdez. Galván, I.; Vacher, M.; Alavi, A.; Angeli, C.; Aquilante, F.; Autschbach, J.; Bao, J. J.; Bokarev, S. I.; Bogdanov, N. A.; Carlson, R. K.; Chibotaru, L. F.; Creutzberg, J.; Dattani, N.; Delcey, M. G.; Dong, S. S.; Dreuw, A.; Freitag, L.; Frutos, L. M.; Gagliardi, L.; Gendron, F.; Giussani, A.; González, L.; Grell, G.; Guo, M.; Hoyer, C. E.; Johansson, M.; Keller, S.; Knecht, S.; Kovačević, G.; Källman, E.; Li Manni, G.; Lundberg, M.; Ma, Y.; Mai, S.; Malhado, J. P.; Malmqvist, P. A.; Marquetand, P.; Mewes, S. A.; Norell, J.; Olivucci, M.; Oppel, M.; Phung, Q. M.; Pierloot, K.; Plasser, F.; Reiher, M.; Sand, A. M.; Schapiro, I.; Sharma, P.; Stein, C. J.; Sørensen, L. K.; Truhlar, D. G.; Ugandi, M.; Ungur, L.; Valentini, A.; Vancoillie, S.; Veryazov, V.; Weser, O.; Wesołowski, T. A.; Widmark, P.-O.; Wouters, S.; Zech, A.; Zobel, J. P.; Lindh, R. OpenMolcas: From Source Code to Insight. Journal of Chemical Theory and Computation 2019, 15, 5925-5964.

(34) Bauschlicher, C. W.; Langhoff, S. R. Full configuration-interaction study of the ionicneutral curve crossing in LiF. The Journal of Chemical Physics 1988, 89, 42464254 .

(35) Roos, B. O.; Taylor, P. R.; Siegbahn, P. E. M. A complete active space SCF method (CASSCF) using a density matrix formulated super-CI approach. Chemical Physics 1980, 48, 157-173.

(36) Dunning Jr., T. H. Gaussian basis sets for use in correlated molecular calculations. I. The atoms boron through neon and hydrogen. The Journal of Chemical Physics 1989, 90, 1007-1023. 
(37) Kendall, R. A.; Dunning Jr., T. H.; Harrison, R. J. Electron affinities of the firstrow atoms revisited. Systematic basis sets and wave functions and wave functions. Journal of Chemical Physics 1992, 96, 6796-6806.

(38) Schäfer, A.; Huber, C.; Ahlrichs, R. Fully optimized contracted Gaussian basis sets of triple zeta valence quality for atoms $\mathrm{Li}$ to Kr. The Journal of Chemical Physics 1994, 100, 5829-5835.

(39) Aquilante, F.; Lindh, R.; Bondo Pedersen, T. Unbiased auxiliary basis sets for accurate two-electron integral approximations. The Journal of Chemical Physics 2007, 127, 114107.

(40) White, S. R. Density matrix formulation for quantum renormalization groups. Physical Review Letters 1992, 69, 28632866. 


\title{
Supporting Information for: Extended
}

\section{dynamically weighted CASPT2: the best of two worlds}

\author{
Stefano Battaglia* and Roland Lindh* \\ Department of Chemistry - BMC, Uppsala University, P.O. Box 576, SE-75123 Uppsala, \\ Sweden
}

E-mail: stefano.battaglia@kemi.uu.se; roland.lindh@kemi.uu.se 


\section{Avoided Crossing in LiF}

In this section we show additional results for the dissociation of lithium fluoride. First, we report the potential energy curves in the case only two states are included in the calculation. The computational details are the same as reported in the main text, with the only difference being the number of roots considered in both the SA-CASSCF and the various CASPT2 calculations. In Figure 1 we report the results of the reference calculation with MRCISD. The results for XDW-CASPT2 for $\zeta=50,5000$ and the limit $\zeta \rightarrow \infty$ are shown in Figures 2

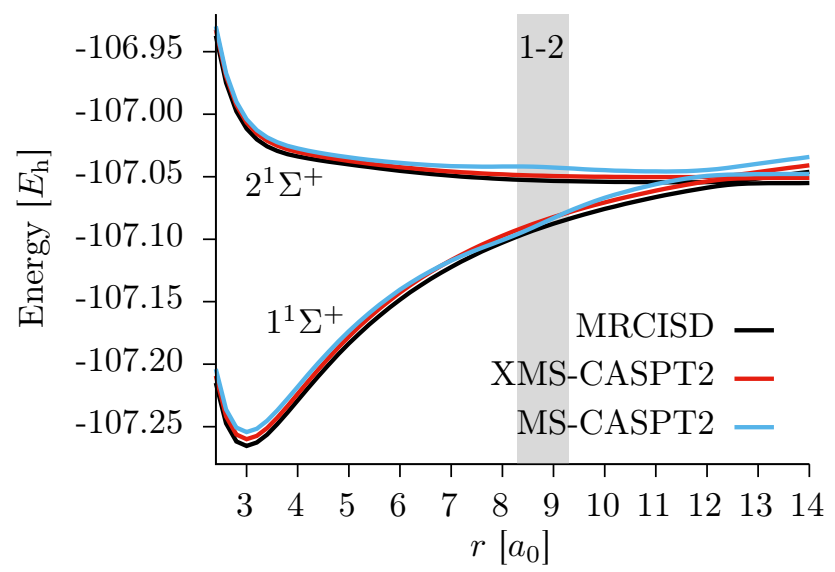

Figure 1: Potential energy curves of the two lowest ${ }^{1} \Sigma^{+}$states of lithium fluoride. The zones highlighted in gray correspond to the avoided crossing regions at the CASSCF level of theory.

to 4. The off-diagonal elements of the Fock operator for $\zeta \rightarrow \infty$ in the three-state case (see main text), are reported in Figure 5. Note that the order of magnitude is the same as the other XDW-CASPT2 calculations presented in the main text. The PECs for $\zeta=500$ are presented in Figure 6 and Figure 7 for calculation with 3 and 2 states, respectively. Density weights and off-diagonal Fock elements are shown in Figures 8 and 9 for the 3-state calculation with $\zeta=500$. 


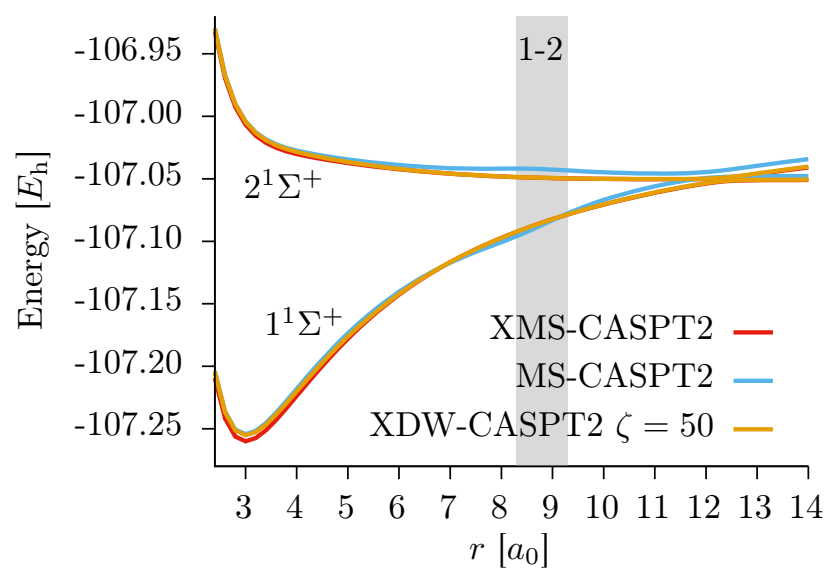

Figure 2: Potential energy curves of the two lowest ${ }^{1} \Sigma^{+}$states of lithium fluoride. Note that to a large extent the XMS-CASPT2 curves are covered by the XDW-CASPT2 ones.

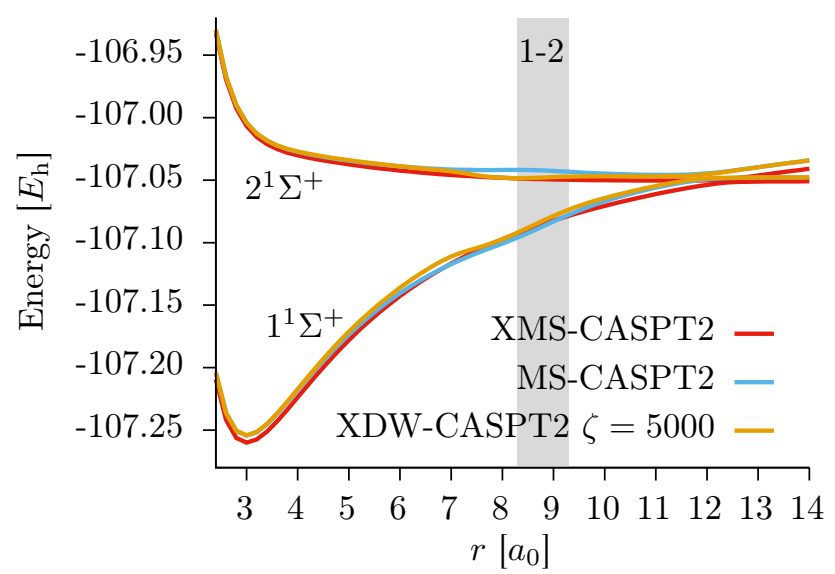

Figure 3: Potential energy curves of the two lowest ${ }^{1} \Sigma^{+}$states of lithium fluoride.

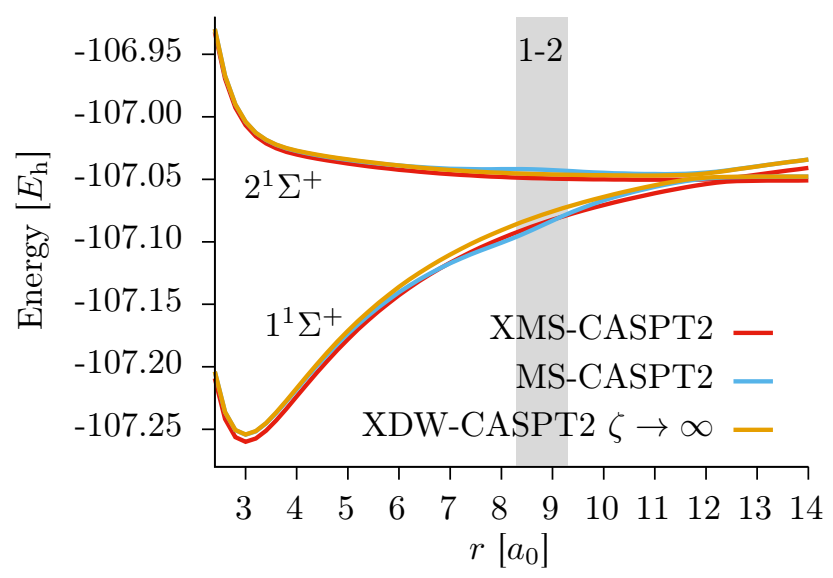

Figure 4: Potential energy curves of the two lowest ${ }^{1} \Sigma^{+}$states of lithium fluoride. 


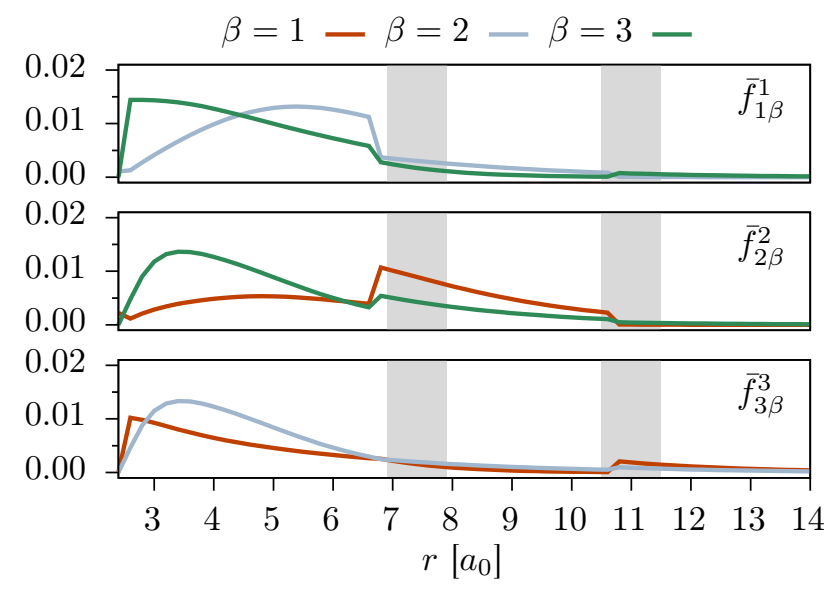

Figure 5: Absolute values of the elements $\bar{f}_{\alpha \beta}^{\alpha}$ for $\zeta \rightarrow \infty$. From top to bottom the couplings are for the ground, first excited and second excited state, respectively. Note that for each state (plot), the Fock operator used to compute the couplings is different.

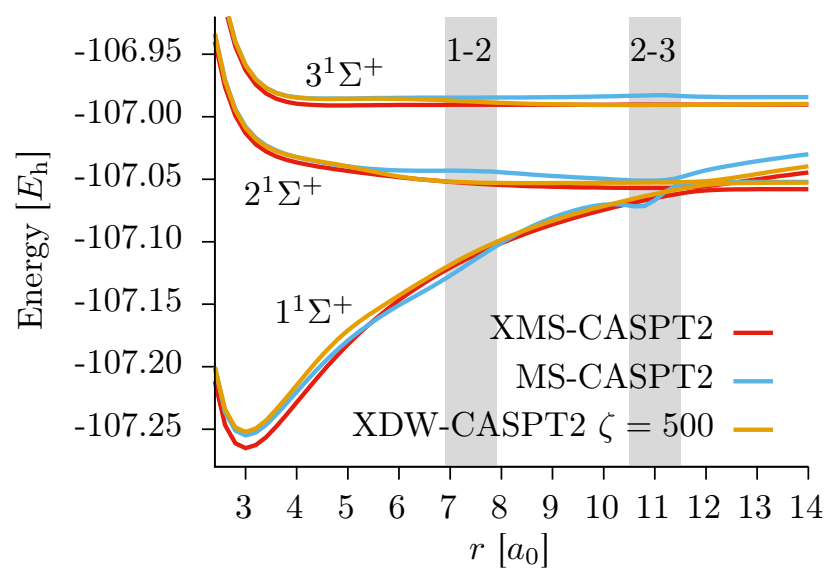

Figure 6: Potential energy surface of the three lowest ${ }^{1} \Sigma^{+}$states of lithium fluoride.

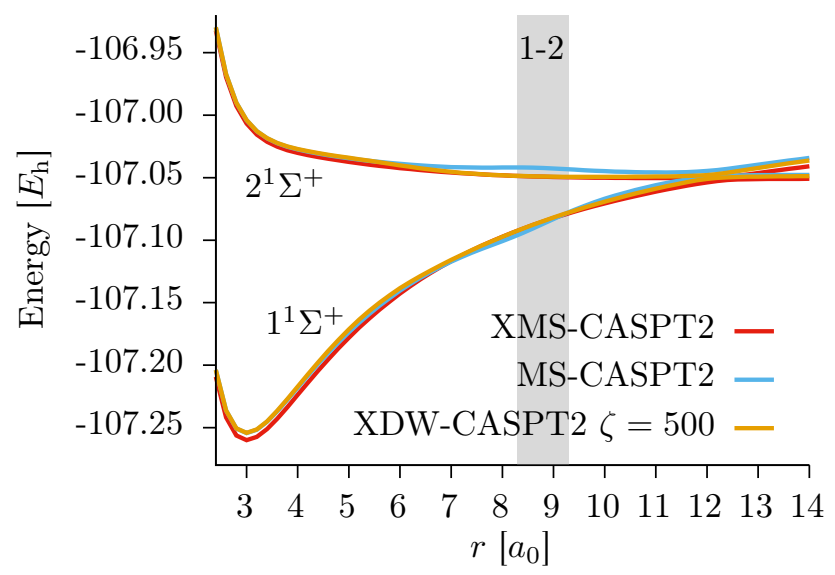

Figure 7: Potential energy curves of the two lowest ${ }^{1} \Sigma^{+}$states of lithium fluoride. 


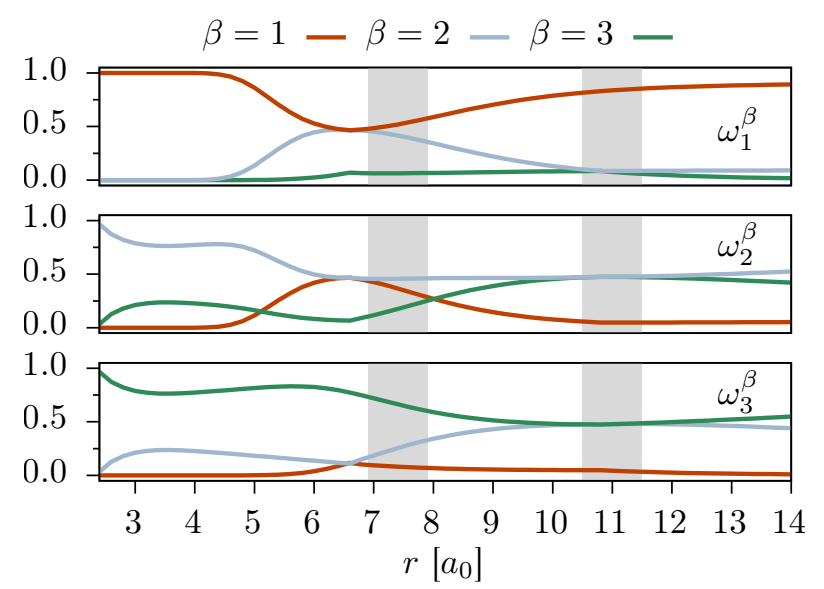

Figure 8: Weights $\omega_{\alpha}^{\beta}$ for $\zeta=500$.

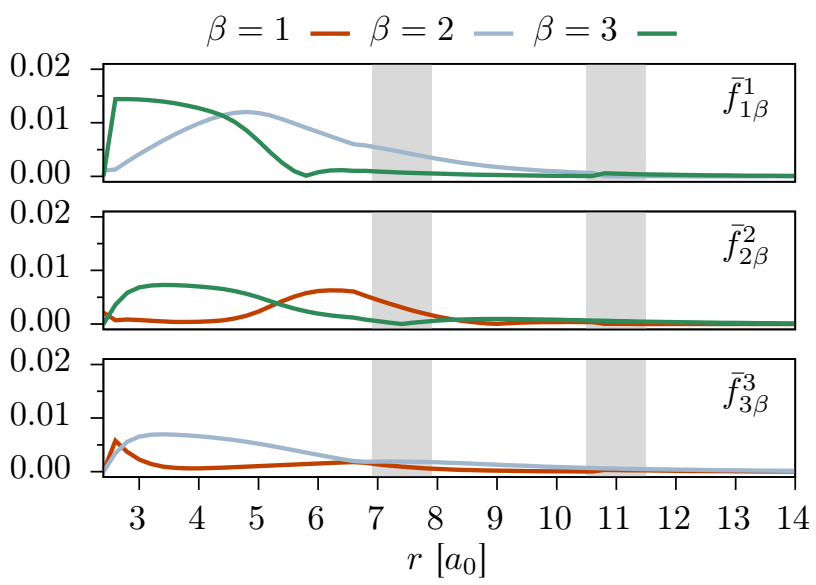

Figure 9: Absolute values of the elements $\bar{f}_{\alpha \beta}^{\alpha}$ for $\zeta=500$. 


\section{Conical intersection of allene}

In this section we report additional computational details of the calculation involving the distorted allene molecule that complement the description in the main text.

The total number of points in the two-dimensional scan is 6561,81 for each variable. The scan of the C-C-C-H torsion angle practically corresponds to a pyramidalization of the

external carbon atoms. Note that in the original work, ${ }^{1}$ the assignment of "variable 1 " and "variable 2" in the surface plots were swapped as compared to the explanation in the text.

The IPEA shift was always set to zero for all methods. For some cases it was necessary to include a real shift in order to converge the calculation for all points. In particular the XMSCASPT2 calculation used a real energy shift of 0.5 Hartree for any size of the model space. The XDW-CASPT2 calculations were performed with a real energy shift of 0.5 Hartree in order to be fairly compared to the XMS-CASPT2 results, even though a smaller shift was sufficient for converge avoiding intruder states.

In order to have visually comparable results to Ref. 1, we plotted the absolute values of the energy difference between the $1^{1} \mathrm{~A}^{\prime}$ and $2^{1} \mathrm{~A}^{\prime}$ states as a colormap with 64 contour iso-surface lines, using a color palette similar to the one of the original work.

In Figure 10 we report the results for a model space with 6 states for XMS-CASPT2. As discussed in the main text, the PES remains virtually unchanged upon increase of the model space dimension. 


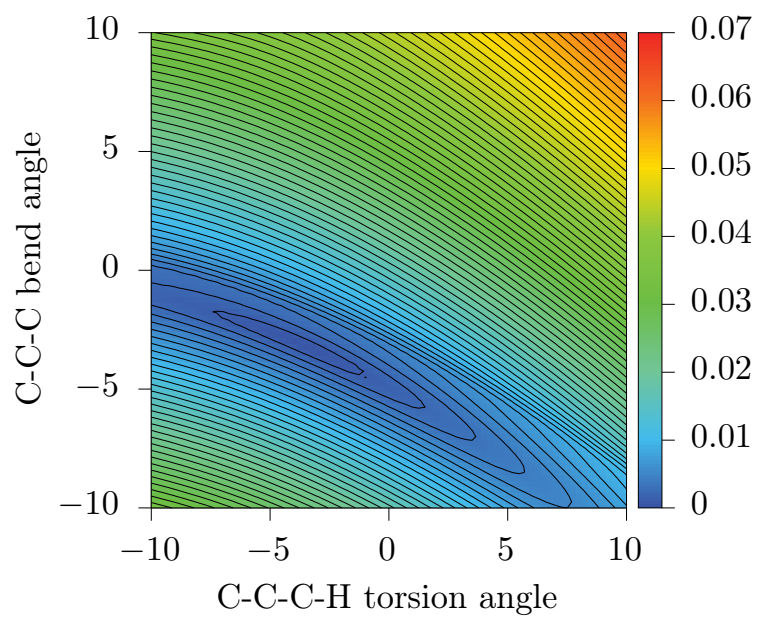

Figure 10: Colormapped isosurface plot of the absolute energy difference (in $E_{\mathrm{h}}$ ) between the $1^{1} \mathrm{~A}^{\prime}$ and $2^{1} \mathrm{~A}^{\prime}$ states for a model space including 6 states. The calculation was carried out with XMS-CASPT2. 


\section{$3 \quad$ Vertical excitation energies}

In this section we report additional information regarding the computational details for the calculation of vertical excitation energies. Imposed molecular point group symmetry, number of active electron, occupied and active orbitals and real denominator shift are listed in Table 1. Note that if a shift was necessary for a method, it was also applied to the other ones in order to obtain comparable results.

Table 1: Computational details of CASSCF and CASPT2 calculations. The string of numbers in the occupied and active orbitals follows the irrep convention of OpenMolcas.

\begin{tabular}{|c|c|c|c|c|c|}
\hline Molecule & PG & $N_{e l}$ & Occ. MOs & Active MOs & Shift \\
\hline acetamide & $\mathrm{C}_{s}$ & 6 & 121 & 13 & 0.1 \\
\hline acetone & $\mathrm{C}_{2 v}$ & 6 & 7411 & 2102 & 0.0 \\
\hline adenine & $\mathrm{C}_{s}$ & 12 & 290 & 010 & 0.1 \\
\hline benzene & $\mathrm{C}_{s}$ & 6 & 180 & 06 & 0.0 \\
\hline benzoquinone & $\mathrm{D}_{2 h}$ & 12 & 80030470 & 031111103 & 0.1 \\
\hline butadiene & $\mathrm{C}_{2 h}$ & 4 & 7006 & 0220 & 0.0 \\
\hline cyclopentadiene & $\mathrm{C}_{2 v}$ & 4 & 9106 & 0220 & 0.1 \\
\hline cytosine & $\mathrm{C}_{s}$ & 10 & 240 & 08 & 0.0 \\
\hline formaldehyde & $\mathrm{C}_{2 v}$ & 4 & 5001 & 0201 & 0.0 \\
\hline formamide & $\mathrm{C}_{s}$ & 6 & 90 & 13 & 0.1 \\
\hline furan & $\mathrm{C}_{2 v}$ & 6 & 9006 & 0320 & 0.0 \\
\hline hexatriene & $\mathrm{C}_{2 h}$ & 6 & 10009 & 0330 & 0.0 \\
\hline imidazole & $\mathrm{C}_{s}$ & 8 & 140 & 15 & 0.1 \\
\hline naphthalene & $\mathrm{D}_{2 h}$ & 10 & 90600707 & 02032030 & 0.2 \\
\hline norbornadiene & $\mathrm{C}_{2 v}$ & 4 & 9635 & 1111 & 0.3 \\
\hline octatetraene & $\mathrm{C}_{2 h}$ & 8 & 130012 & 0440 & 0.0 \\
\hline propanamide & $\mathrm{C}_{s}$ & 6 & 152 & 13 & 0.1 \\
\hline pyrazine & $\mathrm{D}_{2 h}$ & 10 & 50030440 & 12101012 & 0.2 \\
\hline pyridazine & $\mathrm{C}_{2 v}$ & 10 & 9700 & 1133 & 0.0 \\
\hline pyridine & $\mathrm{C}_{2 v}$ & 6 & 11007 & 0420 & 0.2 \\
\hline pyrimidine & $\mathrm{C}_{2 v}$ & 10 & 10600 & 1124 & 0.1 \\
\hline pyrrole & $\mathrm{C}_{2 v}$ & 6 & 9006 & 0320 & 0.0 \\
\hline tetrazine & $\mathrm{D}_{2 h}$ & 14 & 50020340 & 12111112 & 0.1 \\
\hline thymine & $\mathrm{C}_{s}$ & 12 & 270 & 09 & 0.1 \\
\hline triazine & $\mathrm{C}_{s}$ & 12 & 150 & 36 & 0.0 \\
\hline uracil & $\mathrm{C}_{s}$ & 10 & 240 & 08 & 0.0 \\
\hline
\end{tabular}




\section{References}

(1) Granovsky, A. A. Extended multi-configuration quasi-degenerate perturbation theory: The new approach to multi-state multi-reference perturbation theory. The Journal of Chemical Physics 2011, 134, 214113. 